\title{
Utopische Elemente der mittelalterlichen Reiseliteratur
}

\author{
TILO RENZ
}

\begin{abstract}
This article investigates utopian elements in accounts of journeys to the East that circulated in the German-speaking areas in the fourteenth century. The travelogues studied are German translations of the reports of Odoric of Pordenone, Marco Polo and John Mandeville. The paper begins by elaborating on a notion of utopia that can serve as a genre-specific analytical tool for tracing characteristics of early modern utopias in medieval travel literature. At the centre of this notion lies a social structure which significantly differs from the social structure known to the western traveller. Further characteristics complement this concept, particularly those concerning the literary form employed in these descriptions of utopian societies.

The second part of this article will specifically examine the social conditions in the utopian societies presented in the travelogues studied. In addition it will deal with the aesthetic strategies employed by the texts to create specific places, in which kingdoms and other communities with utopian traits are situated in medieval travel literature.
\end{abstract}

Keywords: Travel literature; utopian society; Odoric of Pordenone; Marco Polo; John Mandeville

Im Wortsinn bezeichnet Utopie einen Ort, der an keiner Stelle aufzufinden ist. Eine Paradoxie steht im Zentrum des Begriffs. Der konzeptionelle Wandel von Utopien im Laufe der Zeit, zuallererst aber die widersprüchliche Grundbedeutung des Wortes, hat dazu geführt, dass der Begriff seit dem ersten Erscheinen von Thomas Morus' ,Utopia“ im Jahr 1516 zur Bezeichnung vielfältiger Gegenstände herangezogen worden ist. Wer nach mittelalterlichen Vorformen frühneuzeitlicher Utopien fragt, partizipiert an der Ausweitung des Phänomenbereichs und damit zugleich an einer Veränderung des semantischen Spektrums des Terminus Utopie. Denn nur einzelne Aspekte frühneuzeitlicher Utopien lassen sich bereits in der Literatur der vorausgehenden Jahrhunderte aufweisen.

Um die Veränderungen der Bedeutung von Utopie beim Transfer des Begriffs von der Frühen Neuzeit ins Mittelalter transparent zu machen, ist eine möglichst präzise Bestimmung dessen, was unter Utopie verstanden wird, für jede mittelalterliche Spurensuche zum Phänomen des Utopischen notwendig. Zu Beginn dieses Aufsatzes wird daher ein Verständnis von Utopie entwickelt, mit dessen Hilfe utopische Elemente in der mittelalterlichen Reiseliteratur beschrieben werden können. Als Bezugspunkt dieser Begriffsbestimmung dient neben der mediävistischen Utopie-Forschung vor allem der paradigmatische Text aller frühneuzeitlichen Utopien: Thomas Morus' ,Utopia'. In den folgenden Abschnitten werden unterschiedliche Entwürfe sozial anders geordneter und (zumindest partiell) idealisierter Gemeinschaften in drei mittelalterlichen Reisetexten vorgestellt, die im 14. Jahrhundert im deutschen Sprachraum zirkulierten: im Reisebericht des Odorico da Pordenone, den Konrad Steckel ins Deutsche übertragen hat, in der deutschen Übersetzung des Berichts über Marco Polos Reise nach der Admonter Handschrift sowie im Bericht über die Reisen des Jean de Mandeville in der Übersetzung von Michel Velser. 


\section{Ein Utopie-Begriff für die Reiseliteratur des Mittelalters}

Aus literaturwissenschaftlicher Perspektive hat Tomas ToMAsEK den Versuch einer Begriffsbestimmung der Utopie vorgelegt, die mittelalterliche utopische Entwürfe einschließt. ${ }^{1}$ Danach zeichnet Utopien der Frühen Neuzeit und des Mittelalters aus - lässt man den besonderen Fall des mittelalterlichen Chiliasmus unberücksichtigt ${ }^{2}$-, dass sie „das Wunschbild einer besseren Welt nicht auf das Jenseits verschieben“, sondern ,innerirdische Entwürfe“ dieser Welt anbieten. ${ }^{3}$ Die idealen Gemeinschaften unterscheiden sich in spezifischer Weise von der eigenen: Es handelt sich um ,paradigmatisch andere Welten, die sich nicht mit Einzelheiten oder graduellen Verbesserungen des status quo begnügen“. 4 Mit der spezifischen Form der Andersartigkeit hängt somit eine besondere zeitliche Dimension des Utopie-Begriffs zusammen: Das entworfene Ideal deutet nicht auf die Möglichkeit kurzfristiger Veränderungen der eigenen Lebensumstände hin, sondern es bietet eine „Fernzielorientierung“. 5 Um auf dieses Ziel zu verweisen, haben sich im Mittelalter, so TOMASEK weiter, drei unterschiedliche Modi des Utopischen herausgebildet: die Darstellung einer idealisierten Zeit, eines idealisierten Raums und eines idealisierten Menschen. ${ }^{6}$ Mit dieser Trias schließt TOMASEK an den berühmten Aufsatz ,Wunschräume und Wunschzeiten' an, in dem Alfred Doren bereits in den 1920er-Jahren einen Utopie-Begriff entwickelt hat, mit dem nicht nur frühneuzeitliche, sondern auch antike und mittelalterliche utopische Entwürfe erfasst werden können. ${ }^{7}$ TOMASEK erweitert DoRENs Vorschlag um den Aspekt einer utopischen Anthropologie und er bezieht das Konzept der Wunschräume, das DOREN nur in Antike und Früher Neuzeit realisiert sieht, ${ }^{8}$ auch auf das Mittelalter.

Um utopische Elemente in mittelalterlicher Reiseliteratur herausarbeiten zu können, erscheint es notwendig, einzelne Aspekte von ToMAseKs Utopie-Begriff zu akzentuieren und ihn weiter einzugrenzen. Um deutlich zu machen, dass die Suche nach mittelalterlichen Utopien als Bemühen um die Rekonstruktion einer Vorgeschichte der frühneuzeitlichen so

1 Vgl. Tomas Tomasek, Zur Poetik des Utopischen im Hoch- und Spätmittelalter. Jahrbuch der Oswald von Wolkenstein Gesellschaft 13 (2001/02), S. 179-193.

$2 \mathrm{Zu}$ millenaristischen Vorstellungen, wonach ein ideales Friedensreich zwar auf der Erde, aber erst zum Zeitpunkt der Wiederkehr Christi realisiert wird, vgl. Norman Cohn, The Pursuit of the Millenium. London 1970.

3 Vgl. Tomasek (Anm. 1), S. 179.

4 Ebd., S. 182.

5 Ebd., S. 183.

6 Vgl. ebd.

7 Vgl. Alfred Doren, Wunschräume und Wunschzeiten. Vorträge der Bibliothek Warburg (1924/25), S. 158 -205. Auch andere Forscher haben sich auf Doren bezogen, um einen Begriff des Utopischen zu entwickeln, der auf das Mittelalter angewendet werden kann; vgl. z. B. Ferdinand Seibt, Utopie im Mittelalter. Historische Zeitschrift 208 (1969), S. 555-591, hier S. 556; ders., Utopica. Zukunftsvisionen aus der Vergangenheit. München 2001, S. $13 \mathrm{f}$. (= aktualisierte Neuausgabe von: Utopica. Modelle totaler Sozialplanung. Düsseldorf 1972); Otto Gerhard Oexle, Utopisches Denken im Mittelalter. Pierre Dubois. Historische Zeitschrift 224 (1977), S. 293-339, hier S. 301-305; Gerhard Oexle, Wunschträume und Wunschzeiten. Entstehung und Funktionen des utopischen Denkens in Mittelalter, Früher Neuzeit und Moderne. In: Jörg Calließ (Hg.), Die Wahrheit des Nirgendwo. Zur Geschichte und Zukunft des utopischen Denkens. Rehburg-Loccum 1994, S. 33-83, hier S. 36-39.

$8 \quad$ Vgl. Doren (Anm. 7), S. $182 \mathrm{f}$. 
genannten klassischen Utopien verstanden werden muss und dass die Rekonstruktion daher notwendig an utopische Texte der Frühen Neuzeit als an einen vorläufigen Endpunkt der Entwicklung gebunden bleibt, ziehe ich für die Zuspitzungen und Modifikationen des Utopie-Begriffs Thomas Morus' 'Utopia' als Referenztext heran. ${ }^{9}$

Klassische Utopien zeichnet insbesondere aus, dass in ihnen von räumlichen Vorstellungen anders geordneter Gemeinschaften die Rede ist. Das narrative Muster der Reise ist ein zentrales Merkmal frühneuzeitlicher Utopien. ${ }^{10}$ In ,Utopia' beschreiben zunächst Petrus Aegidius und dann Raphael Hythlodaeus selbst die Expedition, auf der Hythlodaeus auf jene außergewöhnliche Insel getroffen ist. ${ }^{11}$ Das Motiv des Reisewegs in eine anders geordnete Gemeinschaft hat für das Verständnis von Utopien eine zentrale Funktion: Die Schilderung des Wegs an den signifikant andersartigen Ort kann diesen als erfahrbar und damit als existent erscheinen lassen. In diesem Sinne hebt Louis MARIN hervor, dass die Reise als Element der Narration es erlaubt, die utopische Gemeinschaft als Entsprechung der bestehenden Welt darzustellen: „Le récit de voyage nous fait cheminer de l'histoire vers l'utopie. Il nous montre comment un récit peut à la fois se donner comme l'équivalent du monde et comment il peut en composer l'ordre“. ${ }^{12}$ Damit ist das Reiseschema für den epistemischen Status, den ein jeweiliger Text diesem Ort zuweist, von zentraler Bedeutung. ${ }^{13}$ Es schließt den utopischen Ort an die bestehenden Verhältnisse an und kann dabei zugleich den Statuswechsel hin zu einem fingierten Ort markieren. Berücksichtigt man die Verfahren der räumlichen Situierung anders geordneter Gemeinschaften in den so genannten klassischen Utopien, so erscheinen sie nicht einfach als ortlose Orte, sondern werden durch literarische Strategien sowohl konkret verortet als auch entortet. ${ }^{14}$ Thomas NIPPERDEY hat in einer Studie zu frühneuzeitlichen Utopien davon gesprochen, dass diese durch

9 Damit werden die unterschiedlichen Formen, die utopische Entwürfe im 16. und frühen 17. Jh. annehmen können, auf die so genannte humanistische Utopie zugespitzt. Für eine Typologie der Texte vgl. Hans Rudolf Velten, Utopien im 16. Jahrhundert in Deutschland und Europa. In: Werner Röcke u. Marina Münkler (Hgg.), Die Literatur im Übergang vom Mittelalter zur Neuzeit. München, Wien 2004, S. 529-571, hier S. 531-534.

10 Zur Bedeutung des tradierten Musters der Reiseerzählung für frühneuzeitliche Utopien vgl. Micheline Hugues, Émergence de l'utopie. In: Claude Thomasset u. Danièle James-Raoul (Hgg.), En quête d'Utopies. Paris 2005, S. 375-393, hier S. 378.

11 Vgl. Thomas More, Utopia. Hrsg. v. Edward Surtz, S. J. u. Jack H. Hexter (The Complete Works of St. Thomas More, vol. 4). 2. Aufl. New Haven, London 1993, S. 50, 3-52, 24.

12 Louis Marin, Utopiques. Jeux d'espaces. Paris 1973, S. 68.

13 Bedeutsam hierfür ist außerdem die streng rationale Darstellung der besonderen staatlichen Ordnung Utopias: Dass und wie der Staat ohne Privatbesitz funktioniert, wird detailliert und mit großem Bemühen um Plausibilität geschildert (vgl. etwa Miriam Eliav-Feldon, Realistic Utopias. The Ideal Imaginary Societies of the Renaissance 1516-1630. Oxford 1982, S. 2; mit Einwänden: Thomas Schölderle, Geschichte der Utopie. Eine Einführung. Köln u. a. 2012, S. 33-38; zu Vernunft als Fundament des utopischen Staates vgl. bereits Raymond W. Chambers, Thomas More. Harmondsworth 1963, S. 120 f., 252).

14 Das Merkmal ,ohne realen Ort“, mit dem Michel Foucault Utopien charakterisiert, um ihnen Heterotopien an die Seite zu stellen, erfasst die räumlichen Eigenschaften von Utopien nur unzureichend (vgl. Michel Foucault, Von anderen Räumen. In: Jörg Dünne u. Stephan Günzel [Hgg.], Raumtheorie. Grundlagentexte aus Philosophie und Kulturwissenschaften. Frankfurt a. M. 2006, S. 317-329, hier S. 320). Frühneuzeitliche Utopien vereinen in ihrer literarischen Schilderung den Aspekt der konkreten Lokalisierung mit dem der Entortung anders geordneter Gemeinschaften. Ihnen sind beide Merkmale eigen, anhand derer Foucault Utopien und Heterotopien zu unterscheiden versucht. 
die Kategorie der Möglichkeit bestimmt seien, welche sowohl als Potentialität als auch als Irrealität ausgelegt werden könne. ${ }^{15}$ Der Spielraum der Deutungen frühneuzeitlicher utopischer Gemeinschaften zwischen Potentialität und Irrealität wird maßgeblich durch die Relationierung dieser Gemeinschaften zu den bestehenden Verhältnissen und damit durch das Reiseschema und die Darstellung des Raums mitbestimmt, in dem sie situiert werden. Die signifikante Funktion, die der Raumdarstellung somit für die Bedeutung klassischer Utopien zukommt, macht es notwendig, diesem Merkmal auch im Zuge der Untersuchung ferner Utopie-ähnlicher Reiche, von denen die mittelalterliche Reiseliteratur berichtet, besondere Beachtung zu schenken.

Die mediävistische Forschung hat einen Zusammenhang beobachtet zwischen neuen Formen der Fremderfahrung in der Zeit der Kämpfe um Jerusalem im 12. Jahrhundert und dem Aufkommen utopischer Vorstellungen in diesem Zeitraum. ${ }^{16}$ Diese Verbindung festzustellen bedeutet freilich nicht zu behaupten, dass es in zeitgenössischen Beschreibungen von Reisen gen Osten stets um Utopisches geht. Mittelalterliche Reiseschilderungen können aber utopische Elemente enthalten. ${ }^{17}$ Worum es sich dabei im Einzelnen handelt, muss weiter präzisiert werden. Schon mit Blick auf Thomas Morus' ,Utopia“ lässt sich das von TOMASEK zugrunde gelegte Kriterium der paradigmatisch anders geordneten Welt stärker eingrenzen und allein auf das Soziale beziehen. Aus der historisch-zeitgenössischen Perspektive der Europäer, für welche die Teilnehmer des fingierten Gesprächs Raphael Hythlodaeus, Thomas Morus und Petrus Aegidius stehen, zeichnet die Gemeinschaft der Utopier insbesondere aus, nicht auf Privatbesitz, sondern auf Gemeinbesitz gegründet zu sein: haec quamquam essent, ut certe sunt, meliora, tamen aliena uideri possint, quod hic singulorum priuatae sunt possessiones, illic omnia sunt communia. ${ }^{18}$ Nach Einschätzung des Raphael Hythlodaeus ist das Fehlen von Privatbesitz Voraussetzung für eine gerechte und florierende Gesellschaft. ${ }^{19}$ Weil das Interesse des Einzelnen hier mit dem Interesse der Ge-

15 Vgl. Thomas Nipperdey, Die Funktion der Utopie im politischen Denken der Neuzeit. Archiv für Kulturgeschichte 44 (1962), S. 357-378, hier S. 360.

16 Michael Uebel, Ecstatic Transformation. On the Uses of Alterity in the Middle Ages. New York 2005, S. 5 f. knüpft den Beginn des Zirkulierens von Utopie-Entwürfen (z. B. des Briefes des Priesterkönigs Johannes) an das Scheitern des Zweiten Kreuzzugs; vgl. auch Karma Lochrie, Provincializing Medieval Europe. Mandeville's Cosmopolitan Utopia. Publications of the Modern Language Association of America 124 (2009), S. 592-599, hier S. 593; sowie Dietrich Krusche, Utopie und Allotopie. Zur Geschichte des Motivs der außereuropäischen Fremde in der Literatur. Jahrbuch Deutsch als Fremdsprache 11 (1985), S. $131-156$, hier S. 134.

17 Die Unterscheidung zwischen Utopien als Begriff zur Bezeichnung für „das vollständige Ensemble der Sphären und Ebenen einer fiktiven Gesamtgesellschaft“ und den „Elemente[n] dieses utopischen ,Ganzen“" orientiert sich an Richard Saage, Anmerkungen zur Kritik an meinem Plädoyer für das klassische Utopiemuster. In: Ders., Utopisches Denken im historischen Prozess. Materialien zur Utopieforschung. Berlin u. a. 2006, S. 63-78, hier S. 71.

18 Vgl. More (Anm. 11), S. 100, 10-12; Thomas Morus, Utopia. Übers. v. Gerhard Ritter. Stuttgart 2005, S. 51: ,[S]o möchte das [= was die Utopier tun], obgleich es an sich das Bessere wäre - das ist es wirklich - , doch sonderbar erscheinen, weil es hierzulande Privateigentum der einzelnen gibt, dort aber alles Gemeinbesitz ist".

19 Denn wo es noch Privatbesitz gebe, sei ein gerecht geordneter und prosperierender Staat nicht möglich: ubicunque priuatae sunt possessiones, ubi omnes omnia pecunijs metiuntur, ibi uix unquam posse fieri, ut cum Republica aut iuste agatur, aut prospere (More [Anm. 11], S. 102, 21-23). 
meinschaft zusammenfällt, ${ }^{20}$ kümmern sich alle um das allgemeine Wohl. ${ }^{21}$ Die andere soziale Ordnung in Utopia führt also zu anderen Verhaltensweisen der Mitglieder der Gemeinschaft. ${ }^{22}$ Allein Gütergemeinschaft als Grundlage der gesellschaftlichen Ordnung eines utopischen Staates anzusehen, ist als Kriterium allerdings zu strikt, um es auf mittelalterliche Gemeinschaftsentwürfe anwenden zu können. ${ }^{23}$ Dass die Abschaffung von Privatbesitz aber bei Morus als Grundlage der anderen Ordnung der utopischen Gemeinschaft dargestellt wird, legt nahe, bei dem Bemühen um einen Utopie-Begriff, der möglichst eng gefasst ist und sich doch auf mittelalterliche Entwürfe idealisierter Gemeinschaften beziehen lässt, die Andersartigkeit hinsichtlich der sozialen Ordnung als konstitutives Element $\mathrm{zu}$ verstehen. Die folgenden Textanalysen werden zeigen, dass das möglich ist. ${ }^{24}$ Als Teil alternativer sozialer Verhältnisse werden die Zugänglichkeit von Gütern sowie die Besitzverhältnisse, die soziale Schichtung und schließlich die gesellschaftliche Machthierarchie verstanden. Andere Wertvorstellungen und soziale Praktiken können mit diesen sozialen Verhältnissen einhergehen, sie werden hier aber nicht als konstitutiv für mittelalterliche Vorformen von Utopien angesehen.

Weitere Elemente frühneuzeitlicher Utopien, die mit der Bestimmung der anderen sozialen Ordnung als zentralem Bestandteil des Utopie-Begriffs zusammenhängen, müssen auf ihre Anwendbarkeit auf mittelalterliche Entwürfe alternativer Gemeinschaften überprüft werden. So erscheint etwa der Aspekt des Planerischen, den nicht nur TOMASEK erwähnt, ${ }^{25}$ sondern den vor allem SEIBT als zentrales Kriterium mittelalterlicher Utopien ausmacht, ${ }^{26}$ schon bei einem ersten Blick auf die Texte für die Suche nach utopischen Elementen in der Reiseliteratur zu eng zu sein. ${ }^{27}$ Mit der Frage der Planung anderer gesellschaftlicher Organisationsformen ist in weiterem Sinne die Frage der Zeitlichkeit utopischer Entwürfe

20 More (Anm. 11), S. 238, 6-8: Contra hic, ubi omnia omnium sunt nemo dubitat (curetur modo, ut plena sint horrea publica) nihil quicquam priuati cuiquam defuturum; Utopia (Anm. 18), S. 142: „Dagegen hier, wo alles Eigentum Gemeingut ist, zweifelt niemand, daß es keinem für seine Privatbedürfnisse an etwas fehlen wird, solange nur dafür gesorgt wird, daß die öffentlichen Speicher gefüllt sind“".

21 More (Anm. 11), S. 238, 2: Hic ubi nihil priuati est, serio publicum negotium agunt; Utopia (Anm. 18), S. 142: ,[H]ier [...], wo es kein Privateigentum gibt, betreibt man ernsthaft die Interessen der Allgemeinheit".

22 Vgl. auch More (Anm. 11), S. 244, 3-10.

23 Darüber hinaus ist bemerkenswert, dass auch in Morus' ,Utopia' Gemeineigentum nicht zu einer Gesellschaft führt, die in jeder Hinsicht egalitär zu nennen wäre. Seibt, Utopie im Mittelalter (Anm. 7), S. 573 hält treffend fest, dass Utopien zwar zur Gütergemeinschaft tendieren, dass aber „,keine eine wirklich egalitäre Gesellschaft“" entwerfe. In der utopischen Gemeinschaft bei Morus zeigen sich deutlich gesellschaftliche Hierarchien. Sie beruhen auf demokratischer Wahl, Alter, Geschlecht, Verhalten vor dem Gesetz und sozialer Herkunft: Vertreter der Familien werden gewählt; innerhalb der Familie hat das älteste Familienmitglied die Macht; zudem sind Frauen hier den Männern unterstellt; Gesetzesbrecher werden zu Sklaven gemacht; über das Inselreich herrschte ehemals ein König namens Utopus - ob die monarchische Struktur weiterhin Bestand hat, ist unklar (vgl. More [Anm. 11], S. 122, 9; 134, 27f. [vgl. auch 136, $23 \mathrm{f}$.$] ; 136, 24; 184, 17-19; 112, 1$ f.).

24 So auch Heiko Hartmann, Utopias / Utopian Thought. In: Albrecht Classen (Hg.), Handbook of Medieval Studies. Bd. 2. Berlin, New York 2010, S. 1400-1408, hier S. 1400, 1402.

25 Vgl. Tomasek (Anm. 1), S. $179 \mathrm{f}$.

26 Vgl. Seibt, Utopie im Mittelalter (Anm. 7), S. 560; sowie Seibt, Utopica (Anm. 7), S. 11.

27 Oexle hat sich ausdrücklich gegen einen Utopie-Begriff verwahrt, der sich vor allem an der Staats- und Gesellschaftsplanung orientiert, denn diese könne aufgrund anderer Gesellschaftsvorstellungen im Mittelalter nicht angenommen werden (vgl. Oexle, Utopisches Denken [Anm. 7], S. 315 f.). 
angesprochen. Auch räumlichen Utopien der Frühen Neuzeit ist - wie bereits erwähnt eine zeitliche Dimension insofern eigen, als der Modus der Möglichkeit auf eine zukünftige Ordnung der eigenen Gesellschaft verweist. Im letzten Satz der ,Utopia' wird die Zukunftsorientierung deutlich ausgesprochen: ita facile confiteor permulta esse in Vtopiensium republica, quae in nostris ciuitatibus optarim uerius, quam sperarim. ${ }^{28}$ In den Beschreibungen der im Folgenden behandelten mittelalterlichen Utopie-ähnlichen Gemeinschaften scheint die Möglichkeit ihrer zukünftigen Realisierung allenfalls implizit gegeben zu sein.

Ein zentrales Merkmal frühneuzeitlicher Utopien, das in TOMASEKs Ausführungen zum Utopie-Begriff nur knapp Erwähnung findet, ist ihre komplexe formale Gestaltung. ${ }^{29}$ In ,Utopia' berichtet Raphael Hythlodaeus von der idealisierten Gemeinschaft im Rahmen eines Gesprächs, das später, wie es in den Paratexten heißt, von Thomas Morus, einem der Gesprächspartner, lediglich aufgezeichnet und publiziert worden sei. ${ }^{30}$ Die zweifache Übermittlung von Informationen über die utopische Insel weist auf die Ebene des discours (im GENETTE'schen Sinne) hin und damit zugleich auf die Bedeutung ihrer Funktion für die Einschätzung der berichteten Inhalte. Die Mehrstimmigkeit des Gesprächs in ,Utopia“ schafft als literarisches Verfahren Distanz zur beschriebenen Gemeinschaft. ${ }^{31}$ Eine vergleichbar komplizierte Vermittlungsstruktur findet sich unter den utopischen Entwürfen in der mittelalterlichen Reiseliteratur nicht. ${ }^{32}$ Diese Texte distanzieren die geschilderten Gesellschaften in der Regel nicht durch das fortgesetzte Nebeneinander unterschiedlicher Erzählstimmen, die einander kommentieren. Sie betten aber die utopischen Elemente in den Zusammenhang einer Reiseerzählung ein und leisten damit ein gewisses Maß an Rahmung und Perspektivierung. ${ }^{33}$

In der Forschung zu frühneuzeitlichen Utopien ist darüber hinaus vor allem der spielerische Umgang mit Namen als Fiktionalitätssignal gewertet worden. Dazu gehört zunächst der Name der Insel Utopia selbst, der das Eiland gleichermaßen als einen Ort und als

28 More (Anm. 11), S. 246, 1 f.; Utopia (Anm. 18), S. 148: „,[I]ndessen gestehe ich doch ohne weiteres, daß es in der Verfassung der Utopier sehr vieles gibt, was ich in unseren Staaten eingeführt sehen möchte. Freilich ist das mehr Wunsch als Hoffnung“.

29 Vgl. Tomasek (Anm. 1), S. 185.

30 Im Brief an Petrus Aegidius, der in den Ausgaben Louvain 1516 und Basel 1518 enthalten ist, reflektiert Thomas Morus die Schmucklosigkeit seiner Aufzeichnung (vgl. More [Anm. 11], S. 38, 5-14).

31 Zur Betonung der literarischen Verfasstheit von Morus' ,Utopia‘, die in der Forschung spätestens seit den 1960er-Jahren herausgearbeitet worden ist, vgl. den Forschungsüberblick bei Peter Kuon, Utopischer Entwurf und fiktionale Vermittlung. Studien zum Gattungswandel der literarischen Utopie zwischen Humanismus und Frühaufklärung. Heidelberg 1986, S. 11-46. Zur Distanz, die ,Utopia“ zu den behandelten Inhalten schafft, vgl. insbes. Irma Ned Stevens, Aesthetic Distance in the ,Utopia‘. Moreana 11 (1974), S. 13-24.

32 Eine Parallele stellt in den im Folgenden behandelten mittelalterlichen Reisetexten allenfalls das Nebeneinander der Stimmen von Berichterstatter und Übersetzer - etwa die Einschaltungen des Michel Velser in den Text seiner deutschen Übertragung des Jean de Mandeville dar (vgl. Sir John Mandevilles Reisebeschreibung. In deutscher Übersetzung von Michel Velser. Nach der Stuttgarter Papierhandschrift Cod. HB V 86. Hrsg. v. Eric John Morrall. Berlin 1984, 106, 14-16; 123, 16-124, 2; 161, 9-162, 7) - oder die Kombination aus reisendem Berichterstatter und Schreiber - die sich in der deutschen Fassung von Marco Polos Bericht allerdings lediglich auf Andeutungen beschränkt (vgl. Der mitteldeutsche Marco Polo. Nach der Admonter Handschrift. Hrsg. v. Eduard Horst von Tscharner. Berlin 1935, 2, 5-8).

33 Vgl. den Hinweis bei Tomasek (Anm. 1), S. 185. 
nicht-existent ausweist; ${ }^{34}$ dazu gehören aber auch verschiedene andere sprechende Namen von Figuren, wie der des Berichterstatters Raphael Hythlodaeus, ${ }^{35}$ sowie von sozialen Gemeinschaften und Völkern. ${ }^{36}$ Vergleichbares hat die mittelalterliche Reiseliteratur nicht zu bieten.

Ein kompliziertes Gefüge aus Parallelen und Unterschieden zwischen klassischen Utopien und ihren mittelalterlichen Vorformen in der Reiseliteratur ergibt sich aber bei der Darstellung von Räumen. Zumindest in den frühneuzeitlichen Utopien kann sie ebenfalls zu den Mitteln der Markierung von Fiktionalität gezählt werden. Denn die bereits angesprochene Verortung idealisierter Gemeinschaften dient hier nicht nur dazu, sie den realen Verhältnissen anzunähern, sondern die Darstellungen des Raumes enthalten auch Elemente, die diese Gemeinschaften entorten und als fingiert kenntlich machen.

Wie das geschieht, zeigt ein Blick auf die Situierung der Insel Utopia. Der Reiseweg, auf dem Raphael Hythlodaeus das Eiland Utopia erreicht, wird zweimal geschildert: von Petrus Aegidius, einem Freund des Reisenden, in einer auf Ausgangs- und Zielpunkt der Reise beschränkten Reduktionsform ${ }^{37}$ und ausführlicher von Hythlodaeus selbst. ${ }^{38}$ Bereits die Wiederholung verleiht der Darstellung ein gewisses Maß an Komplexität. Die Reise, die Hythlodaeus auch zur Insel Utopia führt, beginnt in einem Kastell, in dem er auf der vierten Expedition des Amerigo Vespucci, welchen Hythlodaeus mehrfach begleitet habe, mit 24 Männern zurückgelassen wird. ${ }^{39}$ Der Verweis auf den Bericht Vespuccis beglaubigt das Geschehen und lokalisiert es an der brasilianischen Ostküste. ${ }^{40}$ Von dort durchfährt der

34 Vgl. etwa R. J. Schoeck, Levels of Word-Play and Figurative Significations in More’s ,Utopia‘. Notes and Queries (1954), S. 512 f., hier S. 512 (,,all [names] share the surface of humor and the deeper signification of unreality“); James D. Simmonds, More's Use of Names in Book II of ,Utopia“. Die Neueren Sprachen 10 (1961), S. 282-284, hier S. 282 f. (,It is readily apparent that no society can be imagined, more antithetical to the real world, than one established nowhere by nobody“); Stevens (Anm. 31 ), S. 18 („The very title [...] suggests the unreality of the island“); Joseph M. Levine, Thomas More and the English Renaissance. History and Fiction in ,Utopia‘. In: Donald R. Kelley u. David Harris Sacks (Hgg.), The Historical Imagination in Early Modern Britain. History, Rhetoric, and Fiction 1500-1800. Washington (DC) 1997, S. 69-92, hier S. 81 (,many of the persons and places are deliberately given names that declare their fictional character").

35 Der Familienname des Berichterstatters, Raphael Hythlodaeus, ist aus griech. v̋ $\theta \lambda$ o $\varsigma$ für ,Geschwätz‘ und

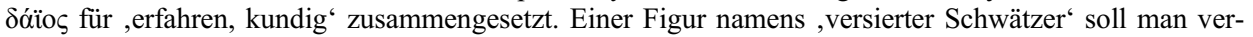
mutlich nicht alles glauben. Die Bedeutung des Namens wird jedoch uneindeutig, wenn man berücksich-

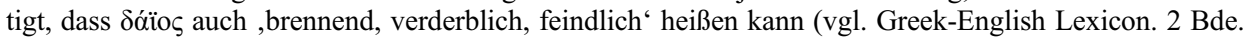
Zusammengest. v. Henry George Liddell u. Robert Scott, rev. u. erw. v. Sir Henry Stuart Jones. Oxford 1948, Bd. 2, S. 1846; Bd. 1, S. 366). Spricht jemand, der so genannt wird, also vielleicht doch ausschließlich die Wahrheit?

36 Zum Beispiel bedeutet der Name der Polyleriten ,viel Unsinn“ und der der Alaopoliten kann mit ,volkloses Volk' übersetzt werden (vgl. More [Anm. 11], S. 74, 21 u. 200, 18; Edward Surtz, S. J. u. Jack H. Hexter, Commentary. In: More [Anm. 11], S. 255-570, hier S. 343 u. 500).

37 Vgl. More (Anm. 11), S. 50, 15-19.

38 Vgl. ebd., S. 50, 25-52, 18.

39 Vgl. ebd., S. 50, 3-9.

40 Über die vier Reisen Vespuccis informiert in aller Kürze der nach dem mutmaßlichen Adressaten so genannte Soderini-Brief, der auf Italienisch erstmals im Zeitraum zwischen 1504 und 1506 veröffentlicht wurde und auf Latein unter dem Titel,Quattuor Navigationes‘ zusammen mit Martin Waldseemüllers ,Cosmographiae Introductio‘ 1507 in St.-Dié erschien (vgl. Die Cosmographiae introductio des Martin Waldseemüller in Faksimiledruck. Hrsg. mit einer Einl. v. Franz R. von Wieser. Straßburg 1907, dort zur 
Reisende viele Länder, kommt schließlich nach Taprobane (Ceylon) und Kalikut an der indischen Südwestküste und wird von portugiesischen Schiffen wieder mit nach Hause genommen. ${ }^{41}$ Wo die Insel Utopia auf dieser weiten Strecke zwischen Brasilien und Indien liegt, bleibt offen; es ist dem Text nicht einmal zu entnehmen, ob die Reisenden in östlicher oder, was zeitgenössisch bereits diskutiert wurde, ${ }^{42}$ in westlicher Richtung nach Indien reisen. In der zweiten, längeren Schilderung des Reisewegs heißt es, sie seien nach vielen Tagen auf Städte und Gemeinschaften gestoßen; ${ }^{43}$ unter ihnen hat sich vermutlich auch die Insel Utopia befunden. Die Entfernung zum Ausgangspunkt, angegeben in vage quantifizierten Tagesreisen, bleibt auch hier unpräzise. Außerdem ist von der äquatorialen Zone die Rede, welche die Reisenden vermutlich passieren, nachdem sie die utopische Gemeinschaft besucht haben. ${ }^{44}$ Dass diese Ortsangaben in exakter Weise unpräzise bleiben, deuten Fiktionalitätssignale an. ${ }^{45}$ Als Markierung von Fiktionalität kann der Hinweis gelten, nur ein wunderbares Glück habe die Reisenden nach Indien und dann zurück nach Europa geführt, ${ }^{46}$ denn Wunderbares wird an anderer Stelle aus dem um streng rationale und gut nachvollziehbare Beschreibung des utopischen Staates bemühten Text ausgeschlossen. ${ }^{47}$ Außerdem behauptet der Erzähler, er habe den Namen des Landes und des Herrschers vergessen, bei dem die Teilnehmer an Vespuccis vierter Expedition zurückgelassen worden sind, ${ }^{48}$ damit provoziert er den Gedanken, dass die Informationen gezielt unterdrückt wurden, um die Lage der utopischen Insel zu verschleiern. ${ }^{49}$ Diese knappen Ausführungen zur Verortung Utopias machen deutlich, dass das Reiseschema in frühneuzeitlichen Utopien nicht nur dazu dient, idealisierte Gemeinschaften in einen realistisch

vierten Reise Vespuccis: S. 97-103; für einen Überblick über die veröffentlichten und unveröffentlichten Briefe Vespuccis vgl. Robert Wallisch, Der Mundus Novus des Amerigo Vespucci. Text, Übersetzung und Kommentar. Wien 2002, S. 123-125.

41 Vgl. More (Anm. 11), S. 50, 15-19.

42 Vgl. Klaus A. Vogel, Sphaera terrae. Das mittelalterliche Bild der Erde und die kosmographische Revolution. Diss. Göttingen 1995, S. 336-383; William G. L. Randles, The Sources of Sir Thomas More's ,Utopia‘. Revista da Faculdade de Letras 22 (1955), S. 157-170, hier S. 159. Die Frage der Westpassage ist zeitgenössisch eng mit der Frage der Gestalt der Erde verknüpft, die insbesondere im Anschluss an die Entdeckung des amerikanischen Südkontinents diskutiert wurde (vgl. dazu knapp Klaus A. Vogel, Cosmography. In: Katharine Park u. Lorraine Daston [Hgg.], The Cambridge History of Science. Bd. 3. Early Modern Science. New York u. a. 2006, S. 469-496, hier S. 476-480).

43 More (Anm. 11), S. 50, 35: post multorum itinera dierum.

44 Vgl. More (Anm. 11), S. 52, 2-7. Dass die Insel tatsächlich südlich des Äquators liegt, macht der Text an anderer Stelle explizit (vgl. More [Anm. 11], S. 196, 29-31).

45 Wie kompliziert es ist, in Morus' ,Utopia‘ Fiktionalitätssignale auszumachen, hat Knape anschaulich gemacht (vgl. Joachim Knape, Fiktionalität und Faktizität als Erkenntnisproblem bei spätmittelalterlichen Reisetexten. In: Holger Krapp u. Thomas Wägenbaur [Hgg.], Künstliche Paradiese - Virtuelle Realitäten. Künstliche Räume in Literatur-, Sozial- und Naturwissenschaften. München 1997, S. 47-62).

46 More (Anm. 11), S. 50, 16: mirabili [...] fortuna.

47 Für das Desinteresse an den Wundern entfernter Länder vgl. More (Anm. 11), S. 52, 29-54, 1.

48 More (Anm. 11), S. 50, 30: (cuius \& patria mihi, \& nomen excidit).

49 Die Unzuverlässigkeit des Berichts ist im Zusammenhang der geographischen Situierung der utopischen Insel ein wiederkehrendes Motiv des Textes. Im Brief des Petrus Aegidius an Hieronymus Busleyden, der ,Utopia' mit anderen Paratexten vorangestellt ist, wird ausführlich erläutert, dass sowohl Morus als auch Aegidius die Angaben des Raphael Hythlodaeus zur Lage der Insel nicht hören konnten, weil sie durch die Nachricht eines Dieners respektive durch das Husten eines Anwesenden gestört wurden (vgl. More [Anm. 11], S. 22, 21-29). 
anmutenden Raum einzubinden. Vielmehr enthält die Beschreibung der Lage der utopischen Insel in Morus' Text darüber hinaus Elemente, die eine genaue Verortung unmöglich machen und die damit anzeigen, dass Utopias Ort ein fingierter ist.

Die folgenden Ausführungen werden unter anderem deutlich machen, wie sozial anders geordnete Reiche in den Reisetexten verortet werden. Vor allem aber konzentrieren sich die Textanalysen auf die Charakterisierung der sozialen Strukturen dieser Länder. Dabei wird in vieler Hinsicht die Nähe zu frühneuzeitlichen Utopien - namentlich zu Morus' ,Utopia“ - deutlich, es treten aber auch Unterschiede hervor.

\section{Utopisches in der mittelalterlichen Reiseliteratur}

Die hier vorgestellten mittelalterlichen Texte berichten von Reisen in den fernen Osten. Sie enthalten Nachrichten über unterschiedliche Gemeinschaften, die mit dem entwickelten heuristischen Utopie-Begriff beschrieben werden können. ${ }^{50}$ Reiseliteratur über den fernen Osten setzt ein mit dem Vordringen der Mongolen nach Europa in der ersten Hälfte des 13. Jahrhunderts. ${ }^{51}$ Zahlreiche Gemeinwesen, die insgesamt oder in Teilaspekten idealisiert erscheinen, werden weit entfernt im Osten verortet, ${ }^{52}$ denn dort befindet sich nach mittelalterlicher Vorstellung das Paradies der Schöpfungsgeschichte. Der wirkmächtigen Augustinischen Genesis-Interpretation folgend wird es $\mathrm{zu}$ allererst historisch verstanden und auf der Erde situiert. ${ }^{53}$ Das irdische Paradies unterscheidet sich von den idealen Orten seiner Umgebung vor allem dadurch, dass es seit dem Sündenfall und der Vertreibung Adams und Evas verschlossen ist. ${ }^{54}$

Alle anderen Länder der fernen Region können Reisende grundsätzlich aufsuchen. Eines von ihnen ist das Reich des Priesterkönigs Johannes, welches als Land des Friedens,

50 Die im Folgenden vorgestellten Gemeinschaften können auch auf die mittelalterliche Erzähltradition über das Schlaraffenland bezogen werden. Für das Schlaraffenland sind soziale Aspekte wie das Fehlen von Privatbesitz und Standesdifferenzen ebenfalls charakteristisch. Vorrangig geht es in diesem Vorstellungskomplex aber um die Bereitstellung sinnlicher Genüsse für die Bewohner - um einen Aspekt also, der in der vorangegangenen Bestimmung des Begriffs Utopie eine nachgeordnete Rolle spielt. Zu Merkmalen des Schlaraffenlands vgl. Nikola Roßbach, Ästhetische Bewältigung von Landlosigkeit. Schlaraffische Perspektiven in Marco Polos und Christoph Kolumbus' Reiseberichten. In: Carsten Jakobi (Hg.), Exterritorialität. Landlosigkeit in deutschsprachiger Literatur. München 2006, S. 15-48, hier S. 23-26; Herman Pleij, Der Traum vom Schlaraffenland. Mittelalterliche Phantasien vom vollkommenen Leben, Frankfurt a. M. 2000, S. 101, 131-133. Zur Aufnahme des Schlaraffenland-Motivs in die mittelalterliche und frühneuzeitliche Reiseliteratur, vgl. Roßbach (Anm. 50), S. $27-48$.

51 Vgl. Gerhard Wolf, Deutschsprachige Reiseberichte des 14. und 15. Jahrhunderts. Formen und Funktionen einer hybriden Gattung. In: Wolfgang Achnitz (Hg.), Deutsches Literatur-Lexikon. Das Mittelalter. Bd. 3. Reiseberichte und Geschichtsdichtung. Berlin, Boston 2012, S. V-XXVIII, hier S. XVI.

52 Berühmtes Gegenbeispiel sind die Paradiesinseln, die in der navigatio- und der Reise-Fassung von Brandans Reisen beschrieben und die im Westen lokalisiert werden.

53 Zur Verortung des Paradieses vgl. Aurelius Augustinus, Über den Wortlaut der Genesis. De Genesi ad litteram libri duodecim. Bd. 2. Übers. v. Carl Johann Perl. Paderborn 1964, Buch VIII, S. 41-85 sowie daran anschließend Thomas von Aquin, Summa theologica. Hrsg. v. P. Heinrich Maria Christmann O. P. München, Heidelberg 1941, 102, 1, S. 173-178. Zur Augustinischen Paradies-Interpretation vgl. auch Reinhold R. Grimm, Paradisus coelestis Paradisus terrestris. München 1977, S. 55-71.

54 Vgl. Monika Unzeitig, Mauer und Pforte. Wege ins Paradies in mittelalterlicher Literatur und Kartographie. Literaturwissenschaftliches Jahrbuch 52 (2011), S. 9-29. 
des materiellen Überflusses und des tugendhaften Verhaltens beschrieben wird. ${ }^{55}$ Seit einer seiner ersten Erwähnungen in der ,Chronica sive Historia de duabus civitatibus' (114346) Ottos von Freising, ${ }^{56}$ also in einem historiographischen Text, ist es vielfach und in unterschiedlichen Textsorten aufgegriffen und tradiert worden. ${ }^{57}$ Jedoch erweisen sich nicht alle Textpassagen der Reiseliteratur, die sich auf die Tradition des Priesterkönigs beziehen, als Darstellungen sozial anders geordneter Gemeinschaften. So ist beispielsweise im Bericht des Franziskaners Johannes von Plano Carpini, welcher im Jahr 1245 im Auftrag des Papstes zu den Mongolen reist, um angesichts der militärischen Bedrohung, die von ihnen ausgeht, mehr über sie zu erfahren, lediglich davon die Rede, dass der Priesterkönig sein Reich wirkungsvoll gegen einen mongolischen Angriff verteidigt hat. ${ }^{58}$ Was das Reich des Presbyters auszeichnet und wie es organisiert ist, wird nicht erwähnt. Der Bericht des Johannes de Plano Carpini zeigt exemplarisch, dass man mit Desinteresse an der utopischen Dimension des Priesterkönigreichs - wie auch eines jeden anderen östlichen Gemeinwesens - rechnen muss. Zugleich weist die Reiseliteratur spätestens seit dem 13. Jahrhundert verschiedene östliche Gemeinschaften mit fremdartig erscheinenden sozialen Ordnungen auf, die in den einzelnen Texten ausführlich behandelt werden. Utopische Elemente in der Reiseliteratur beschränken sich nicht auf einzelne tradierte Motive oder Stoffe, wie das Reich des Presbyters Johannes, sondern sie zeigen sich in einem Spektrum verschiedenartiger ferner Reiche, die zudem in den einzelnen Texten auf ganz unterschiedliche Weise gestaltet sein können.

\section{Der Bericht des Odorico da Pordenone, übersetzt von Konrad Steckel}

Im Jahr 1330 entsteht der Bericht des Franziskaners Odorico da Pordenone über die Asienreise, die er etwa im Zeitraum der vorausgehenden 15 Jahre unternommen hat. ${ }^{59} 1359$

55 Als Utopie hat dieses Reich in der Form, die ihm der vermutlich seit den 1160er-Jahren zirkulierende Brief des Presbyters Johannes gibt, bereits Olschki bezeichnet (vgl. Leonardo Olschki, Der Brief des Presbyters Johannes. Historische Zeitschrift 144 [1931], S. 1-14, hier S. 10-14). Zur Auseinandersetzung mit dieser These vgl. Anna-Dorothee von den Brincken, Presbyter Johannes, Dominus Dominantium - ein Wunsch-Weltbild des 12. Jahrhunderts. In: Anton Legner (Hg.), Ornamenta Ecclesiae. Kunst und Künstler der Romanik. Katalog zur Ausstellung des Schnütgen-Museums. Bd. 1. Köln 1985, S. 8397, hier S. 94 f.; Marina Münkler, Erfahrung des Fremden. Die Beschreibung Ostasiens in den Augenzeugenberichten des 13. und 14. Jahrhunderts. Berlin 2000, S. 192; sowie ausführlich István Bejczy, La lettre du Prêtre Jean. Une utopie médiévale. Paris 2001.

56 Vgl. Otto von Freising, Chronica sive Historia de duabus civitatibus. Hrsg. v. Walther Lammers, übers. v. Adolf Schmidt. Berlin 1960, Buch VII, 33, S. 556-559.

57 Grundlegend zur Tradition vgl. Friedrich Zarncke, Der Priester Johannes. 2 Bde. (Abhandlungen der philologisch-historischen Classe der Königl. Sächsischen Akademie der Wissenschaften 7 u. 8). Leipzig 1879 und 1883; Bettina Wagner, Die Epistola presbiteri Johannis lateinisch und deutsch. Überlieferung, Textgeschichte, Rezeption und Übertragung im Mittelalter. Mit bisher unedierten Texten (MTU 115). Tübingen 2000.

58 Vgl. Giovanni di Pian di Carpine, Storia dei Mongoli. Hrsg. v. Ernesto Menestò. Spoleto 1989, Kap. V, Abschn. 12, S. 258 f. Vgl. auch Gert Melville, Herrschertum und Residenzen in Grenzräumen mittelalterlicher Wirklichkeit. In: Hans Patze u. Werner Paravicini (Hgg.), Fürstliche Residenzen im spätmittelalterlichen Europa. Sigmaringen 1991, S. 9-73, hier S. 33.

$59 \mathrm{Zu}$ Odoricus vgl. Folker Reichert, Einleitung. In: Die Reise des Seligen Odorich von Pordenone nach Indien und China (1314/18-1330). Übers., eingel. u. erl. v. Folker Reichert. Heidelberg 1987, S. 7-20; Reinhold Jandesek, Odoricus von Pordenone. In: Lexikon des Mittelalters. Bd. 6 (1993), Sp. 1362 f. 
schließt der Wiener Weltpriester Konrad Steckel die Übertragung des Textes ins Deutsche ab. ${ }^{60}$ Einleitend stellt der Bericht heraus, dass Odorico die Gegenden, die er schildert, tatsächlich alle bereist habe: ${ }^{61}$ Grundlage seiner Beschreibungen sei an erster Stelle die Augenzeugenschaft; aber auch Informationen von vertrauenswürdigen Personen und schließlich Dinge, die in den besuchten Gegenden zum allgemeinen Wissen gehören, seien aufgenommen worden. ${ }^{62}$

Auch dieser Bericht enthält nur knappe Bemerkungen zum Priesterkönig - in Steckels Übersetzung wird selbst die Erwähnung seines Namens getilgt: Der Herrscher residiere in einer Stadt namens Cosan oder Casan, ${ }^{63}$ die Odorico vom Sitz des Khans von Kathay kommend auf dem Weg nach Westen binnen zwanzig Tagen erreicht habe. ${ }^{64}$ Zahlreiche Städte seien ihm untertan, und er erhalte als Zeichen der engen Verbundenheit mit dem Khan dessen Tochter zur Frau. ${ }^{65}$ Der lateinische Text stellt heraus, dass von den verbreiteten Informationen über den Priesterkönig Johannes nicht einmal der hundertste Teil wahr sei. ${ }^{66}$ In Steckels Übersetzung fehlt diese Bemerkung; sie zeigt aber auch im Positiven wenig Interesse am Presbyter, denn sie nennt - wie bereits erwähnt - nicht einmal den Namen des Herrschers, von dem in diesem Abschnitt die Rede ist.

Ausführlicher schildern lateinischer Text und deutsche Übersetzung das Land Lamori. ${ }^{67}$ Auf dem Reiseweg des Odoricus liegt das Reich 21 Tagesreisen von der vorhergehenden Station entfernt, also vom Land Mobar, einem Landstrich um das Thomas-Grab in Mylapore. ${ }^{68} \mathrm{Im}$ Anschluss an diese im Rahmen eines Itinerars nicht unpräzise Situierung, die lediglich noch um eine Richtungsangabe ergänzt werden könnte, teilt der Berichterstatter mit, die Orientierung verloren $\mathrm{zu}$ haben, und löst Lamori damit nachträglich aus dem

60 Zu Steckels Übersetzung, die im Vergleich zum Bericht des Odoricus etwas kürzer ist, vgl. André Schnyder, Steckel, Konrad. In: Die deutsche Literatur des Mittelalters. Verfasserlexikon. 2. Aufl. Bd. 9 (2010), Sp. 241 -243; sowie Volker Zapf, Steckel, Konrad. In: Deutsches Literaturlexikon. Das Mittelalter. Bd. 3 (2012), Sp. 361-363. Zu Steckels Übersetzung im Kontext der Handschriften, die Odoricos Text überliefern, vgl. Richard Maber u. Angela Tregoning, Conveying the Unimaginable. Odoric of Pordenone's Travels and their vernacular Translations. In: Jean-François Kosta-Théfaine (Hg.), Travels and Travelogues in the Middle Ages. New York 2009, S. 95-134.

61 Für den Text der deutschen Fassung vgl. Konrad Steckels deutsche Übertragung der Reise nach China des Odoricus Pordenone. Hrsg. v. Gilbert Strasmann. Berlin 1968, hier S. 39, 8 f.; für den lateinischen Text vgl. Sinica Franciscana I. Itinera et relationes fratrum minorum saec. XIII et XIV. Hrsg. v. P. Anastasius van den Wyngaert. Florenz 1929, S. 379-495; die Passage, mit der der deutsche Text eröffnet, steht in der lateinischen Fassung am Schluss (vgl. Odoricus, Wyngaert [Anm. 61], Kap. 38, 6, S. 494); dort wird nicht eigens erwähnt, dass Odorico alle Orte, von denen er berichtet, selbst bereist hat.

62 Vgl. Steckel (Anm. 61), S. 39, 5-9; sowie Odoricus, Wyngaert (Anm. 61), Kap. 38, 6, S. 494.

63 Die Stadt ist nicht klar zu identifizieren; vgl. Paul Pelliot, Notes on Marco Polo. Bd. 2. Paris 1963, S. $813 \mathrm{f}$.

64 Vgl. Steckel (Anm. 61), 117, 827 und 119, 865-867; sowie Odoricus, Wyngaert (Anm. 61), Kap. 32, 1, S. 483 (hier wird die Entfernung mit 50 Tagesreisen angegeben).

65 Vgl. Odoricus, Wyngaert (Anm. 61), Kap. 32, 1, S. 483; sowie Steckel (Anm. 61), 119, 867-869.

66 Vgl. Odoricus, Wyngaert (Anm. 61), Kap. 32, 1, S. 483: de quo non est centesima pars eius quod quasi pro certo dicitur de ipso.

67 Vgl. Steckel (Anm. 61), 63, 220-65, 237; sowie Odoricus, Wyngaert (Anm. 61), S. 445 f. Es handelt sich um eine Region im Nordosten der Insel Sumatra, die auch Lambri genannt wird (vgl. Pelliot [Anm. 63] Bd. 2, S. $761 \mathrm{f}$.).

68 Vgl. Steckel (Anm. 61), 63, 220-222; sowie Folker Reichert, Erläuterungen. In: Reise des Seligen Odorich von Pordenone (Anm. 59), S. 136. 
räumlichen Zusammenhang heraus, der durch die Schilderung des Reisewegs entsteht. ${ }^{69}$ Das Land zeichnet sich durch Gemeinbesitz an Grund und Boden sowie an Frauen und Kindern aus. ${ }^{70}$ Allein die Häuser sind in privatem Besitz. ${ }^{71}$ Es herrscht kein Mangel an wertvollen und daher repräsentativen Gütern sowie an grundlegenden Nahrungsmitteln, denn die Natur stellt diese Güter in ausreichender Menge zur Verfügung: An aller speiß, prót, vleisch, guet gold, holcz, aloe vnd camphora vnd vil andrer edler ding wéchst da genúg. ${ }^{72}$

Dass die Menschen auf Kleidung verzichten, wird mit der großen Hitze begründet, die in ihrem Land herrscht. ${ }^{73}$ Der Berichterstatter bewertet dieses besondere Verhalten selbst nicht, sondern er erzählt umgekehrt davon, dass er von den Fremden verspottet wird, weil er bekleidet geht. ${ }^{74}$ Sie berufen sich darauf, dass Gott Adam nackt erschaffen habe. ${ }^{75}$ Damit werden die Fremden zusätzlich idealisiert, denn ihr Verhalten wird mit der menschlichen Daseinsform vor dem Sündenfall verständlich zu machen gesucht. Sie üben Kritik an den zeitgenössischen Christen des Westens, indem sie daran erinnern, dass sich deren Verhalten vom eigentlich idealisierten ursprünglichen Zustand weit entfernt hat. Die Kritik aus der Perspektive der vermeintlich Fremden wird erkennbar innerhalb des christlichen Horizonts formuliert. Die Begegnung mit den Fremden erhält so die Funktion, grundlegende christliche Idealvorstellungen (hier die Möglichkeit eines Lebens ohne Scham und damit einer paradiesischen Situation) zu artikulieren. Als Vorlage für diese Konstellation hat vermutlich die Gymnosophisten-Episode gedient, die aus der Literatur über Alexander den Großen bekannt ist. ${ }^{76}$ Indem der Bericht über das Land Lamori die Darstellung eines idealisierten Reiches mit der Funktionalisierung dieser Darstellung für die Kritik an bestehenden Verhältnissen in Mitteleuropa verbindet, weist er zwei Merkmale auf, die sich in frühneuzeitlichen Utopien $\mathrm{zu}$ einem grundlegenden Strukturelement der Darstellung herausbilden: In Thomas Morus' ,Utopia‘ schlägt sich diese Verbindung in der Gliederung des Textes in zwei Bücher nieder, von denen das erste der Kritik an den zeitgenössischen Verhältnissen in England gewidmet ist und das zweite den eigentlichen Entwurf des idealen Staates enthält. Gleichwohl bleibt die Kritik in Odoricos Bericht deutlich im mittelalterlichen Horizont, denn sie zielt mit dem prälapsalen Zustand als Korrektiv auf ein Ideal, dessen Realisierung nach christlicher Vorstellung nicht in der Macht der Zeitgenossen ste-

69 Vgl. Steckel (Anm. 61), 63, 222 f. Die Desorientierung wird in Steckels Fassung zugespitzt; im lateinischen Text ist lediglich davon die Rede, dass der Erzähler an diesem südlichen Punkt den Polarstern nicht mehr sehen könne und deshalb offenbar nicht in der Lage ist, sich innerhalb des Landes Lamori zu orientieren: in qua incepi amittere tramontanam, cum terra michi acceperat eam (Odoricus, Wyngaert [Anm. 61], S. 445).

70 Vgl. Steckel (Anm. 61), 63, 226-230.

71 Vgl. ebd., 63, $230 \mathrm{f}$.

72 Ebd., 63, 232-234.

73 Vgl. ebd., 63, $223 \mathrm{f}$.

74 Vgl. Steckel (Anm. 61), 63, 224-226.

75 Ebd., 63, $225 \mathrm{f}$.

76 Vgl. Friedrich Pfister, Das Nachleben der Überlieferung von Alexander und den Brahmanen. Hermes 76 (1941), S. 143-169; Karl Stackmann, Die Gymnosophisten-Episode in deutschen Alexander-Erzählungen des Mittelalters. Beiträge zur Geschichte der Deutschen Sprache und Literatur 105 (1983), S. 331353. 
hen kann. ${ }^{77}$ In ,Utopia ${ }^{6}$ dagegen wird zu plausibilisieren versucht, wie durch die besondere Einrichtung des Staates bestimmte Laster, die den Menschen seit dem Sündenfall eigen sind, korrigiert werden konnten. ${ }^{78}$

Noch eine weitere Eigenart des Landes Lamori unterscheidet diese Gemeinschaft deutlich von jenen, über die in frühneuzeitlichen Utopien die Rede ist. Trotz des allgemeinen Reichtums, der partiellen Gütergemeinschaft und den Parallelen zur Situation vor dem Sündenfall wird das Land dennoch nicht idealisiert. Bei den Bewohnern handele es sich um gar vnrain leẃt, denn sie würden sich von Menschenfleisch ernähren. ${ }^{79}$ Darunter sind auch die Kinder anderer Völker, die Händler ihnen zum Kauf anbieten. ${ }^{80}$ Eigenschaften, die das Land Lamori als idealen Lebensraum erscheinen lassen und die zur Kritik der Verhältnisse im Westen führen, gehen also mit deutlich negativ bewerteten Charakteristika dieses Landes einher.

\section{Marco Polos Reisebericht in deutscher Fassung}

Etwa dreißig Jahre vor dem Bericht über die Reise des Odoricus von Pordenone ist bereits Marco Polos Reiseschilderung entstanden. Dieser Text ist im deutschen Sprachraum in der Folgezeit zunächst nicht weit verbreitet: Zwei deutschsprachige Übersetzungen sind in wenigen Handschriften und frühen Drucken überliefert. ${ }^{81}$ Die erste Übertragung ins Deutsche, die im Laufe des 14. Jahrhunderts verfasst wird, enthält in der Einleitung die Behauptung, Marco Polo habe das Beschriebene autoptisch erfahren. ${ }^{82}$ Dass er viele Dinge dieser Welt mit eigenen Augen gesehen habe, zeichne ihn vor anderen Menschen aus, denn niemand habe so weite Reisen unternommen wie er; die Wahrheit des Geschilderten wird dabei ausdrücklich an die Anwesenheit vor Ort gebunden. ${ }^{83} \mathrm{Im}$ Folgenden nennt der Text mehrfach Marco Polo als erlebendes Ich. ${ }^{84}$ Die deutsche Übersetzung stützt die Geltung des Geschilderten damit vor allem auf die Erfahrung des Reisenden. ${ }^{85}$

77 Der Rekurs auf die spezifische Situation vor dem Sündenfall wird deutlich, auch wenn die Fremden von dieser Phase der Heilsgeschichte allgemeine Handlungsanweisungen Gottes ableiten: got hiet Adam nakchatn geschepht vnd jch trúeg gewant an wider gotz ardnung (Steckel [Anm. 61], 63, 225 f.).

78 Vgl. More (Anm. 11), S. 244, 2-13; vgl. auch Eliav-Feldon (Anm. 13), S. 81-83.

79 Steckel (Anm. 61), 63, 231 f. Kannibalismus zählt zu den Stereotypen fremder Völker, die aus der Antike ins Mittelalter übernommen werden (vgl. John Block Friedman, The Monstrous Races in Medieval Art and Thought. Syracuse 2000, S. 10, 26-29, 116; Claude Lecouteux, Les monstres dans la littérature allemande du moyen âge. Bd. 2. Göppingen 1982, S. 9 f.; vgl. auch Rudolf Simek, Erde und Kosmos im Mittelalter. Das Weltbild vor Kolumbus. München 1992, S. 105-107).

80 Vgl. Steckel (Anm. 61), 63, 234-65, 237.

81 Vgl. Ernst Bremer, Polo, Marco. In: Die deutsche Literatur des Mittelalters. Verfasserlexikon. 2. Aufl. Bd. 7 (2010), Sp. $771-775$, hier Sp. $774 \mathrm{f}$.

82 Marco Polo (Anm. 32), 1, 12 f.: also her selbe [= Marco Polo] spricht das her mit sinen ougin geseen habe.

83 Marco Polo (Anm. 32), 1, 13-15: Ouch helt er das vor wor das keyn mensche von Adams geczitin habe also vil gewandirt [...] und also manchirleyge geseen an der worheit [Hervorhebung T. R.]. Schwer zu untermauern ist die These von Ertzdorffs, die Übertreibung schränke die Glaubwürdigkeit der Beteuerung ein (vgl. Xenja von Ertzdorff, Marco Polos ,Beschreibung der Welt‘ im 14. und 15. Jahrhundert in Deutschland. In: Gerhard Augst [Hg.], Festschrift für Heinz Engels zum 65. Geburtstag. Göppingen 1991, S. 46-63, hier S. 49).

84 Vgl. etwa Marco Polo (Anm. 32), 54, 25; 57, 18; 69, 15.

85 Die vermutlich älteste franko-italienische Fassung F gesteht dagegen zu, dass Marco Polo einiges, von dem die Rede sei, nicht selbst gesehen, sondern von vertrauenswürdigen Personen gehört habe (vgl. Marco Polo, Il Milione. Prima edizione integrale a cura di Luigi Foscolo Benedetto. Florenz 1928, Abschn. 1, S. 3, 8-12). 
Auch in diesem Text wird das Reich des Priesterkönigs Johannes erwähnt, der in der deutschen Fassung von Marco Polos Bericht Preami heißt. ${ }^{86}$ Wo der Herrschaftsbereich des Presbyters liegt, wird nicht erläutert, und auch über die besondere Organisation des Landes informiert der Text nicht. Erwähnt wird nur die Größe des Reiches, und es wird geschildert, wie der Priesterkönig den Tartaren in einer Schlacht unterlegen ist, die im Jahr 1187 stattgefunden haben soll, und dass er dadurch seine Vormachtstellung verliert.

Im engeren Sinne utopische Züge trägt dagegen das Land Mangi, das wie Lamori bei Odorico da Pordenone an das Reich der Brahmanen der Alexander-Tradition erinnert. Es handelt sich offenbar um den südlichen Teil Chinas, jenseits des Gelben Flusses. ${ }^{87}$ Das Volk des Landes ist nicht waffenfähig, sondern gibt sich dem Müßiggang und den weltlichen Genüssen hin. ${ }^{88}$ Dieses Verhalten hängt offenbar mit einer Furchtlosigkeit zusammen, die sich aus der sehr allgemein beschriebenen Macht des Landes und aus der Befestigung der Städte ergibt. ${ }^{89}$ Über die Kühnheit des Herrschers macht der Text widersprüchliche Angaben: Er wird als reich, kampffähig und machtvoll eingeführt und danach doch als verweichlicht bezeichnet. ${ }^{90}$ Als oberstem Richter gelingt es ihm, einen Rechtszustand zu etablieren, der allgemein akzeptiert wird: Die Bewohner des Landes lassen regelmäßig ihre Häuser unverschlossen, ohne dass ihnen dadurch Schaden entsteht. ${ }^{91}$ Sowohl die unbestrittene Machtposition des Reiches als auch der innere Friedenszustand zeigen, dass die Lebensbedingungen den Mitgliedern der Gemeinschaft ein sorgloses Leben ermöglichen. Außerdem beweist der Herrscher seine Barmherzigkeit mit der regelmäßigen Fürsorge für Waisenkinder. ${ }^{92}$ Die Passage unterscheidet zwischen dem König und den Bewohnern des Landes, enthält aber keine Hinweise auf eine weitere soziale Differenzierung. Der beschriebene ideale Zustand der Gemeinschaft ist offenbar nicht von Dauer, denn im folgenden Abschnitt berichtet der Text, dass das Land vom Großen Khan unterworfen worden ist. ${ }^{93}$

Die deutsche Fassung von Marco Polos Reisebericht beschreibt ferner das Reich Mulete des Alten vom Berge. ${ }^{94}$ Die historische Vorlage hierfür bilden die religiöse Gemeinschaft der Ismailiten oder Assassinen sowie ihr berühmtester Führer Rašīdaddīn Sinān (1163-1193). ${ }^{95}$ Bei Marco Polo wird dieses Reich unpräzise als Station auf dem Reiseweg

86 Vgl. insbes. Marco Polo (Anm. 32), 15, 21 -16, 14; für den Begriff Preami vgl. 15, 23.

87 Marco Polo (Anm. 32), 39, $25 \mathrm{f}$.: und wen man kumt obir das wassir [= Cakomoya, vgl. Marco Polo (Anm. 32), 39, 19], so kumt man in eyne groze provincien Mangi genant. Zum Reich Mangi vgl. auch Pelliot (Anm. 63) 1, S. 274 f. Zum Gelben Fluss, der hier mit einer Variante des mongolischen Namens Caramoran bezeichnet wird, vgl. Pelliot (Anm. 63) Bd. 1, S. $182 \mathrm{f}$.

88 Vgl. Marco Polo (Anm. 32), 39, $31 \mathrm{f}$.

89 Vgl. ebd., 39, $32 \mathrm{f}$.

90 Vgl. ebd., 39, 29 f. und 40, 3.

91 Vgl. ebd., 40, $3 \mathrm{f}$.

92 Vgl. ebd., 40, 4-10.

93 Vgl. ebd., 40, 11-41, 2.

94 Vgl. ebd., 10, 15-11, 17. Zu Mulete, dem islamischen und chinesischen Namen der Ismailiten, vgl. Pelliot (Anm. 63) Bd. 2, S. $785-787$.

95 Vgl. M. C. Lyon, Assassinen. In: Lexikon des Mittelalters. Bd. 1 (1980), Sp. 1118 f.; Leonardo Olschki, Marco Polo's Asia. An Introduction to his ,Description of the World' Called ,Il Milione‘. Berkeley, Los Angeles 1960, S. 362-381; Fahrad Daftary, The Assassin Legends. Myths of the Isma'ilis. London, New York 1995, S. 67-84, 101-118. Hier ist noch nachzutragen, dass auch Odorico von Pordenone eine Passage zum Gebiet des Alten vom Berge bringt (vgl. Steckel [Anm. 61], 127, 929-129, 959), das 
situiert; die Entfernung zum vorhergehenden Ort bleibt unbestimmt, die zum folgenden wird jedoch angegeben. ${ }^{96}$ Die Besonderheit dieses Reiches besteht in Marco Polos Schilderung in einem künstlich angelegten, verschlossenen Garten, welcher, wie es im Text heißt, der Paradiesvorstellung des Islam nachgebildet ist. ${ }^{97}$ Seine Elemente versprechen sinnliche Genüsse: prachtvoll geschmückte Bauten, Früchte, außerdem vier Flüsse aus Wasser, Wein, Milch und Honig sowie Jungfrauen, die singen und musizieren. ${ }^{98}$ Gemacht ist dieses Paradies für junge Männer, die dem Garten von außen zugeführt werden. Der Alte vom Berge setzt die Jünglinge mit Hilfe des Gartens für seine politischen Ziele ein. Sie werden durch ein Getränk mit magischer Wirkung, einen tolyn trank, ${ }^{99}$ eingeschläfert und in das künstliche Paradies getragen; dort erleben sie lust mit den jungen Frauen; ${ }^{100}$ nach einiger Zeit werden sie erneut betäubt und wieder herausgebracht. Das durch die Prozedur implementierte Begehren der Einzelnen nach dem zuvor erlebten, vermeintlich paradiesischen Zustand kann anschließend als Machtmittel eingesetzt werden: do sprach her, wer do wolle kumen in das paradys, der muste ym syn undirtenik. [...] do vant man vil di durch begerunge des paradys gingen in den tod unde mortin alle di Velgius gebot czu totin. ${ }^{101}$ Die menschlichen Waffen bedeuten einen Machtgewinn für den Alten, denn zahlreiche Mächtige der Region werben um seine Gunst. Aber auch der Herrscher über Persien habe von dieser bosheit erfahren, ${ }^{102}$ die Burg belagert und schließlich den Alten und sein Gefolge umgebracht. ${ }^{103}$ Die abschließende negative Bewertung des künstlichen Paradieses ist deutlich. Nicht in erster Linie aufgrund der Markierung als Gemeinschaft muslimischen Glaubens, ${ }^{104}$ sondern vor allem aufgrund der Ausrichtung auf die machtpolitischen Interessen eines einzelnen wird dieses Paradies als soziale Einheit in seiner Idealität massiv eingeschränkt. Diejenige unter den hier vorgestellten anders geordneten Gemeinschaften, bei der der Aspekt der Planung hervortritt, wird damit abschließend negativ bewertet. Auch die innere Ordnung des künstlichen Paradieses unterscheidet sich von den bereits beschriebenen Utopie-ähnlichen Reichen bei Marco Polo und in anderen Reisetexten: Im Garten herrscht ein genussvoller Zustand; es stellt sich aber keine soziale Gemeinschaft her, denn der Zugang zum künstlichen Paradies sowie alles, was darin geschieht, wird von einem Einzelnen kontrolliert, der allein in der Lage ist, die Grenze zwischen dem abgegrenzten, alternativ organisierten Ort und seiner Umgebung willentlich zu überschreiten. Mit dem

in der Übersetzung Steckels Jmlestore heißt (vgl. Steckel [Anm. 61], 127, 929); Odoricos Beschreibung stimmt in allen wesentlichen Details mit der Marco Polos überein (vgl. auch Daftary [Anm. 95], S. 117 f.).

96 Vgl. Marco Polo (Anm. 32), 11, $15 \mathrm{f}$.

97 Vgl. ebd., 10, 24-27. Vgl. Der Koran. Übers. v. Ahmad Milad Karimi. Freiburg i. Br. u. a. 2009, Sure 56, 1-40, Sure 78, 31-34. Die Besonderheit von Marco Polos Beschreibung des Reiches des Alten vom Berge besteht darin, dass er ihm Züge des Paradieses verleiht (vgl. Daftary [Anm. 95], S. 113).

98 Vgl. Marco Polo (Anm. 32), 10, 17-20; 10, 24-28.

99 Ebd., 10, 30.

100 Ebd., 11, 1.

101 Ebd., 11, 5-9. Diese Form des organisierten, politisch motivierten Mords, die mit uneingeschränktem Gehorsam einem Meister gegenüber einhergeht, löst die Faszination der Zeitgenossen Marco Polos für die Assassinen aus (vgl. Olschki [Anm. 95], S. 366-381).

102 Marco Polo (Anm. 32), 11, 10.

103 Vgl. ebd., 11, 10-15.

104 Vgl. ebd., 10, $23 \mathrm{f}$. 
Reich Mangi verbinden diesen Ort das sorglose Leben, die sinnlichen Genüsse und das spezifische zeitliche Verhältnis zur Gegenwart des Erzählens: Als über beide Gemeinschaften berichtet wird, existieren sie schon nicht mehr.

\section{Die Reisen des Jean de Mandeville in der Übersetzung des Michel Velser}

Mitte des 14. Jahrhunderts entsteht der Bericht über die Reisen Jeans de Mandeville; in den 1390er Jahren wird er von Otto von Diemeringen und von Michel Velser ins Deutsche übertragen. Auf dessen Übersetzung beziehen sich die folgenden Ausführungen. ${ }^{105} \mathrm{Im}$ ersten Teil schildert der Text eine Pilgerfahrt ins Heilige Land, und die Einleitung kündigt zunächst auch nur diesen Inhalt des Buches an. ${ }^{106}$ Erst die knappe Übersicht über das Folgende am Schluss des einleitenden Kapitels macht deutlich, dass der Reisebericht auch von Ländern handeln wird, die weit östlich von Jerusalem liegen. ${ }^{107}$ Am Ende der Einleitung behauptet der Erzähler von sich, alles, was er beschreibe, mit eigenen Augen gesehen zu haben: Und von landen und ynselen wil ich úch sagen, so ich úmer ebnost kan, wie sie gestalt sind, und als ich das alles selb gesenhen hon. ${ }^{108}$ Die Forschung des 19. Jahrhunderts hat gezeigt, dass das mitnichten der Fall gewesen ist, sondern dass es sich bei Mandeville um einen so genannten armchair traveller gehandelt hat. ${ }^{109}$ Vor allem die Reiseberichte des Wilhelm von Boldensele und des Odorico da Pordenone dienten als Quellen für die zwei Teile des Textes. ${ }^{110}$ Hier sollen weder die Plagiats-Vorwürfe der Forschung des 19. Jahrhunderts wiederholt noch die besonderen literarischen Qualitäten von Mandevilles Text herausgearbeitet werden, um ihn auf dieser Grundlage zu würdigen. ${ }^{111}$ Interessant für

105 Ich zitiere im Folgenden aus: Sir John Mandevilles Reisebeschreibung. In deutscher Übersetzung von Michel Velser. Nach der Stuttgarter Papierhandschrift Cod. HB V 86. Hrsg. v. Eric John Morrall. Berlin 1984. Zu Besonderheiten und Überlieferung dieser Fassung vgl. Ernst Bremer, Mandeville, Jean de (John, Johannes von). In: Die deutsche Literatur des Mittelalters. Verfasserlexikon. 2. Aufl. Bd. 5 (1985), Sp. 1201-1214, hier Sp. $1208 \mathrm{f}$.

106 Der erste Satz lautet: Do ich von hayman $u ß$ für in den müt und in der sach das ich wolt faren úber mer zu dem hailigen grab und zu dem gesegnotten ertterich, das man in lattin nempt Terra Promissionis (Mandeville, Velser [Anm. 105], 1, 4-6).

107 Vgl. ebd., 3, 11-23.

108 Ebd., 3, 23 f. Kurz darauf folgt der Hinweis, dass er die Reise mit einigen zuverlässigen Begleitern unternommen habe (vgl. ebd., 4, 1 f.).

109 Vgl. Albert Bovenschen, Untersuchungen über Johann von Mandeville und die Quellen seiner Reisebeschreibung. Zeitschrift der Gesellschaft für Erdkunde zu Berlin 23 (1888), S. 177-306, hier S. 200 f., 206-306.

110 Die These, Mandeville habe den Asien-Teil von Odorico da Pordenone übernommen, vertritt bereits Henry Yule, Cathay and the way thither. Medieval notices of China. Bd. 1. London 1866, S. $27 \mathrm{f}$. Zusammenfassend zu Mandevilles Quellen vgl. Bremer (Anm. 105), Sp. $1204 \mathrm{f}$.

111 Vgl. vor allem den Versuch der Rehabilitierung Mandevilles durch Josephine Waters Bennett, The Rediscovery of Sir John Mandeville. New York 1954; vgl. aber auch noch Mary B. Campbell, The Witness and the Other World. Exotic European Travel Writing 400-1600. Ithaca 1988, insbes. S. 141 (,he [= Mandeville] shaped a fiction"); Klaus Ridder, Werktyp, Übersetzungsintention und Gebrauchsfunktion. Jean de Mandevilles Reiseerzählung in deutscher Übersetzung Ottos von Diemeringen. In: Xenja von Ertzdorff u. a. (Hgg.), Reisen und Reiseliteratur im Mittelalter und in der frühen Neuzeit. Vorträge eines interdisziplinären Symposiums vom 3.-8. Juni 1991 an der Justus-Liebig-Universität Gießen. Amsterdam u. a. 1992, S. 357-388, insbes. S. 364 (,die Art der Quellenverarbeitung im einzelnen und die Struktur des Werkganzen [erweist] die Darstellungsintention des Autors als eine literarische“). 
die Frage nach utopischen Elementen scheint vielmehr die spezifische Konzeption von Wissen über andere Länder und Regionen zu sein, die der Text enthält und die bereits in der Einleitung zum Ausdruck kommt. ${ }^{112}$ Der Erzähler schränkt hier die Vollständigkeit und Verlässlichkeit seiner Angaben ein, und rechnet mit der Aktivität der Rezipienten. Das unterscheidet diesen Text von den Berichten Odoricos da Pordenone und Marco Polos.

Bereits der zitierte Satz, in dem der Erzähler behauptet, alles, wovon er berichte, selbst gesehen zu haben, weist auf die Grenzen der Informationen hin: Er beschreibe ferne Länder, sofern er dazu in der Lage sei (so ich úmer ebnost kann). ${ }^{113}$ Was die Akkumulation von Wissen über die Fremde begrenzt hat, bleibt offen. ${ }^{114}$ Am Schluss der Einleitung steht die Aufforderung an die Leser, die Wahrheit des Textes zu prüfen ([sie] múgen verniemen ob ich war sag oder lieg) und Fehler gegebenenfalls zu korrigieren: ob ich an kainer lay sach falte, daz sie das recht machtend; und weiter: Dar umb wil ich, das yederman, wa ich nit recht hette, mich múge gestrauffen. ${ }^{115}$ Die kritische Rezeption wird offenbar nicht nur erwartet, sondern sogar gefordert. ${ }^{116} \mathrm{Zu}$ dieser ausdrücklich formulierten Orientierung am Rezipienten gehört der zweimalige Hinweis, dass der Text in der Volkssprache geschrieben sei, damit eine größere Zahl von Menschen ihn verstehen könne. ${ }^{117}$ In diesem Zusammenhang ist auch die Mehrstimmigkeit der deutschen Fassung durch Michel Velser zu berücksichtigen: An verschiedenen Stellen meldet sich der Übersetzer zu Wort und bestätigt, die Angaben des Textes aus seinem Wissenshorizont. ${ }^{118}$ Diese Einschübe stützen nicht einfach nur die Angaben des Textes, sondern sie sind zugleich ein Hinweis auf die in der Einleitung geforderte Praxis der Auseinandersetzung mit diesen.

Unter den Regionen des Ostens, die im zweiten Teil des Textes beschrieben werden, ${ }^{119}$ sind verschiedene Reiche, die utopische Züge tragen. Unter anderem schildert Mandeville hier das Priesterkönigreich des Johannes. Seine Darstellung ist vergleichsweise ausführlich - zwei Erzählsequenzen sind dem Priesterkönig gewidmet ${ }^{120}$-, und umfasst auch die so-

112 Bereits Greenblatt würdigt in seiner Mandeville-Interpretation das Wie der Darstellung nicht nur in seiner literarischen Faktur, sondern er fragt auch nach dessen Effekten auf das dem Text inhärente Wissen (Stephen Greenblatt, Marvellous Possessions. The Wonder of the New World. Chicago 1991, S. 26-51, insbes. S. 46-51). Mit der Frage nach dem Verhältnis von Sprache und Welt fokussiert Greenblatt allerdings einen anderen Aspekt, als ich im Folgenden betonen werde.

113 Mandeville, Velser (Anm. 105), 3, $23 \mathrm{f}$.

114 Offenbar handelt es sich nicht um eine Grenze der durch den Text transportierten Information, welche die Form der Darstellung vorgibt - von ihr ist kurz darauf die Rede (vgl. Mandeville, Velser [Anm. 105], 4, 16 f.) -, sondern um Wissenslücken der Erzählinstanz.

115 Ebd., 4, 8; 4, 8f.; 4, $10 \mathrm{f}$.

116 Campbell weist auf die entsprechende Stelle hin, nimmt sie aber nicht als Information über den Status der Aussagen des Textes ernst, sondern wertet sie als Teil des elaborierten literarischen Spiels, das sie Mandeville unterstellt; zudem deutet sie unter Hinzuziehung weiterer Textstellen die Aufrufe des Textes zur Skepsis gegenüber den eigenen Aussagen sehr spitzfindig als impliziten Ausschluss des Zweifels an seiner Glaubwürdigkeit (vgl. Campbell [Anm. 111], S. 144-148).

117 Vgl. Mandeville, Velser (Anm. 105), 3, 8-10 und 4, 3-7.

118 Vgl. ebd., 106, 14-16;123, 16-124, 2; 161, 9-162, 7; sowie weitere Zusätze der zweiten Redaktion des Textes.

119 Vgl. ebd., 93, 1-178, 20.

120 Vgl. ebd., 153, $21-157,24 ; 170,10-26$. 
ziale Organisation des Reiches. ${ }^{121}$ Der Weg dorthin ist sehr weit und gefährlich und führt durch Kathay. ${ }^{122}$ Zumindest Teile des Priesterkönigreichs sind wegen wilder Tiere so schwer zugänglich, dass der Erzähler selbst nicht dorthin gelangt ist. ${ }^{123}$ [G] ${ }^{\circ}$ tter fryd und grosser richtung zeichnen das Land aus. ${ }^{124}$ Reichtum und ein allgemeiner Friedenszustand wirken sich auf die Lebensverhältnisse aller Bewohner aus. Dennoch scheint die hierarchische Strukturierung der Gesellschaft dadurch nicht aufgelöst zu werden; denn zumindest von einer Differenzierung des Adels in Könige, Herzöge und Grafen ist die Rede. ${ }^{125}$ Am Presbyter selbst wird gezeigt, dass die Reichtümer sich auf das Verhalten der Menschen positiv auswirken: Wertvolle Materialien, insbesondere Edelsteine, fördern die Tugenden des Herrschers. Sein Bett aus Saphir vertreibt die Unkeuschheit. ${ }^{126}$ Die Mahlzeiten nimmt der Priesterkönig gemeinsam mit einer großen Menschenmenge nur einmal am Tag ein was auf die Partizipation vieler am Reichtum des Herrschers ebenso hindeutet wie auf verbreitete Selbstdisziplin und Enthaltsamkeit. ${ }^{127}$

$\mathrm{Zu}$ den weiteren Themen der Passage über den Priesterkönig zählt die Religion: Ein Großteil der Bewohner sei christlichen Glaubens, dieser weiche aber in seinen Inhalten durchaus vom westlichen Christentum ab. ${ }^{128}$ Außerdem ist von zahlreichen wundern die Rede, wie zum Beispiel dem steinernen Fluss oder einem Feld, auf dem im Laufe eines jeden Tages Pflanzen empor wachsen und wieder vergehen. ${ }^{129}$ Auch von den Herrschaftszeichen, die der Priesterkönig im Kriegsfalle und bei nicht-militärischen Ausritten und Reisen verwendet, wird berichtet. ${ }^{130}$ Die Passage macht deutlich, dass sich der Friedenszustand, der zuvor benannt worden ist, nur auf die Situation innerhalb des Landes bezieht und nicht auf die Interaktion mit anderen Herrschaftsräumen.

Die zweite ausführlichere Sequenz über den Priesterkönig Johannes berichtet davon, wie er seinen Namen erhalten hat: ${ }^{131}$ Er sei nach Alexandria gereist, um zu erfahren, wie Christen den Gottesdienst feiern; dort in der Kirche sei er zufällig Zeuge einer Priesterweihe geworden; verwundert über den Vorgang habe er nachgefragt (es duht in gar wunderlich, und fräget ${ }^{132}$ ) und auf die Antwort hin entschieden, sich nach dem ersten der Priester, der aus der Kirche trete, zu nennen; dieser habe Johannes geheißen: ich wil och priester haissen, und wil das man mich nemme als der erst priester haisset der uß der kirchen gat.

121 In diesem Sinne hat Melville den ,,diesseitigen Charakter“ von Mandevilles Schilderung des Priesterkönigreichs herausgestellt (vgl. Melville [Anm. 58], S. 61).

122 Vgl. Mandeville, Velser (Anm. 105), 154, 11; 154, 14 f.; 154, 20-23. Zusätzlich zur Situierung des Priesterkönigreichs jenseits des Reiches des Khans von Kathay bietet der Text noch eine weitere Art der Verortung an (154, 23-28): Man erreiche Indien, indem man Persien durchquere, bis zu einer Stadt namens Hermospole gelange und von da übers Meer bis zur Stadt Cabat reise; dort angekommen, könne man dann noch weiter reisen - in welcher Richtung und wie lange, bleibt allerdings offen.

123 Vgl. ebd., 170, 4-8.

124 Ebd., 154, 9.

125 Vgl. ebd., 157, 14-17.

126 Vgl. ebd., 157, 7-10.

127 Vgl. ebd., 157, 10-22.

128 Vgl. ebd., 155, 5-8.

129 Vgl. ebd., 155, 10-156, 5.

130 Vgl. ebd., 156, 5-17.

131 Vgl. ebd., 170, 10-26.

132 Ebd., 170, $18 \mathrm{f}$. 
Und der priester hieß Johans. ${ }^{133}$ Die Episode zeigt, dass der Name Priesterkönig Johannes nicht nur Zeichen des Respekts dieses Herrschers für den christlichen Glauben und seines Anspruchs auch auf religiöse Führerschaft ist. Vielmehr steht dieser Name in der kurzen Passage auch für die Aufgeschlossenheit gegenüber dem, was aus der Perspektive des indischen Herrschers weit im Westen liegt, für seine Neugier und für seine Bereitschaft zur Übernahme von Elementen einer fremden Kultur. Dass der Presbyter nicht die Priesterweihe anstrebt, sondern nur den Namen des Priesters tragen will, zeigt die Modifikation westlicher kultureller Praktiken im Zuge ihrer Adaption durch den östlichen Herrscher an. ${ }^{134}$ Nicht nur in seiner Macht, seinem Reichtum und seiner Tugendhaftigkeit scheint der Priesterkönig hier idealisiert zu werden, sondern auch in seiner Wissbegier und Aufnahmebereitschaft für Fremdes. Auch hierin kann eine Parallele zu den Utopien der Frühen Neuzeit gesehen werden: Raphael Hythlodaeus berichtet vom regen Interesse der Utopier an der Kultur der Europäer, und er vertritt sogar die Auffassung, dass Utopia vor allem deshalb vernünftiger regiert werde als die europäischen Staaten, weil die Europäer nicht bereit seien, von anderen zu lernen und Institutionen zu übernehmen, die anderswo besser organisiert sind als bei ihnen. ${ }^{135}$

Die ynsel Bragine, die dem Reich der Brahmanen der Alexander-Tradition entspricht, ${ }^{136}$ liegt in nicht näher bestimmter Nachbarschaft eines anderen Königreichs im Land des Khans von Kathay, welches der Text nicht durch einen Namen kenntlich macht. ${ }^{137}$ In diesem Land wächst alles, was den Bewohnern zum Leben notwendig ist. ${ }^{138}$ Privilegiert sind die Menschen dort außerdem, weil ihnen manche Unannehmlichkeiten erspart bleibt: Es gibt keinen Tod, weder Hagel, noch Donner und Blitz, keinen Krieg, keinen Hunger und keine Krankheiten. ${ }^{139}$ Mit den erleichterten Lebensbedingungen geht außerordentlich tugendhaftes Verhalten der Bewohner der Insel einher. Sie hüten sich vor Bosheit und Sündhaftigkeit; ${ }^{140}$ ausdrücklich erwähnt wird, dass sie Hoffart und Unkeuschheit nicht kennen; ${ }^{141}$ außerdem halten sie die zehn Gebote - sind also offenbar christlichen Glaubens und achten Reichtum gering. ${ }^{142}$ Weitere Tugenden folgen (Frömmigkeit, Gerechtigkeit,

133 Ebd., 170, 21-23.

134 Man kann also mit Burkes Typologie des kulturellen Austauschs von selektiver Aneignung sprechen; Burke hat auf die Thematisierung dieser in der Frühen Neuzeit wieder auflebenden Form der kulturellen Interaktion bereits bei den Kirchenvätern hingewiesen (vgl. Peter Burke, Kultureller Austausch. Frankfurt a. M. 2000, S. 17 f.).

135 Vgl. More (Anm. 11), S. 108, 14-19.

136 Vgl. Mandeville, Velser (Anm. 105), 175, 5. In einer anschließenden Einschaltung des Übersetzers, Michel Velser, wird die Verbindung zum Alexander-Stoff explizit gemacht.

137 Ebd., 174, 24: Und by der ynsel da ist ain ander güt ynsel. Mandeville übernimmt die Episode von Odorico da Pordenone, bei dem das Land Mançi oder Manzi heißt (vgl. Odoricus, Wyngaert [Anm. 61], Kap. 34, 1 f., S. 486-488; Steckel [Anm. 61], 125, 911-127, 928); es wird in Oberindien lokalisiert (vgl. Pelliot [Anm. 63] Bd. 2, S. 750).

138 Vgl. Mandeville, Velser (Anm. 105), 174, 24.

139 Vgl. ebd., 175, $14 \mathrm{f}$;; 175, $22 \mathrm{f}$. Was mit der Abwesenheit von Tod (sterbend) genau gemeint ist (ebd., 175, 14), wird nicht recht deutlich, denn das Leben der Menschen dort ist - wie es an anderer Stelle heißt - zwar lang, aber nicht unbegrenzt (vgl. ebd., 175, 22 f.).

140 Vgl. ebd., 174, 25.

141 Vgl. ebd., 174, 26.

142 Vgl. ebd., 174, 27-175, 1; von der Missachtung des Reichtums ist später erneut die Rede (vgl. ebd., 176, 2). 
kein Diebstahl, keine Lüge, kein Mord, kein Ehebruch), ${ }^{143}$ und es wird erwähnt, dass die Menschen auf der Insel Bragine fasten und nur nach Maßgabe ihrer dringendsten Bedürfnisse essen und trinken. ${ }^{144}$ Diese besondere Tugendhaftigkeit sei den in Mitteleuropa bekannten Verhaltensweisen entgegengesetzt. ${ }^{145}$ Verantwortlich für die europäischen Verhältnisse sei schlicht die Sündhaftigkeit: das ist unser súnd schuld. ${ }^{146}$ Das sündfreie Verhalten der Menschen in Bragine dagegen sei Gott wohlgefällig, und es habe vermutlich, so spekuliert der Erzähler, zu den besonderen Lebensbedingungen in diesem Land geführt: $D a$ von ist es [= die zuvor erwähnten Lebensbedingungen] wol ain zaichen das sie gott lieb hat. ${ }^{147}$ Das Ideal der sündenlos handelnden Fremden wird herangezogen, um die Sündhaftigkeit der eigenen Gesellschaft zu kritisieren. Anders als in Morus' ,Utopia“ - und anders auch als es Mandevilles Schilderung des Priesterkönigreichs des Johannes nahelegt - wird tugendhaftes Verhalten dabei nicht von besonderen gesellschaftlichen Verhältnissen abhängig gemacht, sondern der Entscheidung des einzelnen unterstellt.

In der Schlusspassage des Abschnitts bezieht sich der Text explizit auf die Begegnung Alexanders des Großen mit den Brahmanen. Die Passage gibt Aufschluss über die Gesellschaftsstruktur des Reiches: Das Kollektiv der Bewohner (sie) spricht den makedonischen Herrscher an. ${ }^{148}$ Einen König hätten sie nur, um sich anderen Herrschaftsverbänden ähnlich zu machen; eine Funktion, beispielsweise die der Rechtsprechung, komme ihm nicht $\mathrm{zu}$, denn es gebe in ihrem Land keine Konflikte, die gerichtlich entschieden werden müssten. ${ }^{149}$ Über diesen funktionslosen König hinaus finden sich keine weiteren Hinweise auf eine Binnendifferenzierung der Gemeinschaft. Der Abschnitt über das Reich der Brahmanen in Mandevilles Bericht enthält zudem weder explizit markierte wunderbare Elemente noch Dystopisches. Er deutet auch nicht auf ein Interesse der Bewohner an den Fremden hin, sondern zeigt lediglich, dass sie ihr Wissen an andere weitergeben.

Irgendwo unweit vom Sitz des Priesterkönigs Johannes liegt das Land Pasamsore oder Millestorrach. ${ }^{150}$ Das ist der Name, den Mandeville dem Reich des Alten vom Berge gibt. Die Darstellung stimmt weitgehend mit der Schilderung bei Marco Polo und in Steckels Übersetzung des Berichts von Odorico da Pordenone überein. ${ }^{151}$ Anders als bei diesen wird das Reich hier jedoch schon eingangs negativ bewertet: Der Ort wird beherrscht von einem Zauberer, der waz bosser den der túffel. ${ }^{152}$ Auch dass das Reich nicht mehr existiert, wird bereits zu Beginn der Passage deutlich, ${ }^{153}$ abschließend wird es noch einmal erwähnt mit dem Hinweis, dass immer noch erkennbar sei, wo ehemals das künstliche Paradies gewesen ist. ${ }^{154}$ Das vergangene Reich hat im Raum Spuren hinterlassen. Mandeville betont die wunderbaren Elemente: Schmuck und Inventar des Palastes, Brunnen, Tiere und Jungfrauen. Auch von der Art und

143 Vgl. ebd., 175, $10 \mathrm{f}$.

144 Vgl. ebd., 175, 21 f.; 175, 13.

145 Ebd., 175, 16: das kumpt uns alles widerwätig.

146 Ebd., 175, 17.

147 Ebd., 175, $17 \mathrm{f}$.

148 Ebd., 175, 25.

149 Vgl. ebd., 176, 10-14.

150 Ebd., 158, 1: Nit verre von in dem land da ist ain ynsel.

151 Vgl. Marco Polo (Anm. 32), 10, 15-11, 17 und Steckel (Anm. 61), 127, 929-129, 959; s. o., S. 142 f.

152 Mandeville, Velser (Anm. 105), 158, 5.

153 Vgl. ebd., 158, $4 \mathrm{f}$.

154 Vgl. ebd., 159, $17 \mathrm{f}$. 
Weise, auf die die jungen Männer instrumentalisiert werden, ist die Rede, ${ }^{155}$ es fehlt allerdings der Hinweis auf Morde an zeitgenössischen Herrschern - statt dessen ist davon die Rede, dass der Zauberer ihnen befiehlt, sich selbst umzubringen ${ }^{156}$ - und auf die Machtposition, die der Schöpfer des künstlichen Paradieses auf diese Weise erlangt.

Auch von der Insel Lamori, die schon Odorico da Pordenone beschreibt, berichtet Mandeville. ${ }^{157}$ Die Angaben zur Verortung des Landes sind bei Mandeville ausführlicher: Auch hier wird es zur Region um das Thomas-Grab an der Ostküste Südindiens in Relation gesetzt; ${ }^{158}$ weiter präzisierend heißt es: Lamori liege auf einer Insel etwa 52 Tagesreisen seewärts vor dieser Küste. ${ }^{159}$ Es gebe dort keine Scham, keine eheliche Bindung zwischen Mann und Frau und auch der Besitz scheint grundsätzlich allen verfügbar zu sein. ${ }^{160}$ Dieser Aspekt wird betont, indem der Text anders als Steckel in seiner Übersetzung des Berichts von Odorico da Pordenone nicht auf die Grenze des Gemeinbesitzes aufmerksam macht (die Häuser jedoch seien Privatbesitz, heißt es dort), sondern das gemeinschaftliche Eigentum zu einer Gleichheit an Reichtümern zuspitzt: Und sind all gelich rich ${ }^{161}$ Lamori ist damit sozial weitgehend egalitär organisiert. Allerdings wird auch hier - wie in Steckels Odorico-Übertragung - die Idealität des Landes eingeschränkt durch den abschließenden Hinweis auf den Kannibalismus, der bei den Bewohnern üblich sei. ${ }^{162}$

\section{Ergebnisse und weiterführende Perspektiven}

Der Durchgang durch die drei Texte hat deutlich gemacht, dass sozial anders geordnete Gemeinschaften in der mittelalterlichen Reiseliteratur auf verschiedenartige Weise gestaltet sein können und dass ihre besondere soziale Organisation mit einer Reihe weiterer Charakteristika einhergeht. Die Gemeinwesen kennen beispielsweise privates Eigentum nicht, wie das Land Lamori vorführt (bei Odorico da Pordenone und Jean de Mandeville); ihre Bewohner leben in einem Zustand des Friedens und der materiellen Sorglosigkeit, wie das Land Mangi (bei Marco Polo) und das Priesterkönigreich des Johannes (bei Jean de Mandeville) zeigen; Reichtum wirkt sich positiv auf die Tugendhaftigkeit des Verhaltens der Einzelnen aus, und eine Hierarchie der Gesellschaft ist lediglich im Amtstitel des Herrschers realisiert, wie das Land Bragine deutlich macht (bei Jean de Mandeville); Exponenten der Gemeinschaften erweisen sich als außerordentlich wissbegierig in Bezug auf Fremdes, wie der Priesterkönig Johannes exemplifiziert (ebenfalls bei Jean de Mandeville); oder sie erleben in Abgeschiedenheit von der Welt eine kurze Zeit der vielfältigen sinnlichen

155 Vgl. ebd., 159, 6-14. Das Begehren nach dem Paradies als Ursache für die Gefügigkeit der jungen Menschen wird hier stark verkürzt als Effekt des Zaubertranks dargestellt und nicht, wie bei Marco Polo und in Konrad Steckels Übersetzung des Berichts von Odorico da Pordenone, durch den Entzug des bereits erfahrenen künstlichen Paradieses (vgl. Steckel [Anm. 61], 129, 940-949; Marco Polo [Anm. 32], 11, 2-9).

156 Mandeville, Velser (Anm. 105), 159, 13.

157 Vgl. ebd., 112, 11-113, 10.

158 Vgl. ebd., 109, 14-16; s. o., S. 139.

159 Vgl. ebd., 112, 13-15.

160 Vgl. ebd., 112, 15-21; 112, 22-113, 3.

161 Ebd., 113, 3.

162 Vgl. ebd., 113, 4-10. Wie bei Odorico wird diese Praxis deutlich abgewertet durch den Hinweis, dass in dem Land genug Nahrungsmittel verfügbar sind (vgl. Mandeville, Velser [Anm. 105], 113, 5 f.). 
Genüsse, wie die jungen Männer, die der Alte vom Berge für sein künstliches Paradies rekrutiert (bei allen drei Autoren).

Es fällt auf, dass die behandelten sozial anders geordneten Gemeinschaften nicht durchweg idealisiert, sondern unterschiedlich bewertet werden. Wertungen durch die Erzählinstanz können, wie die Schilderung des Reiches des Alten vom Berge bei Marco Polo zeigt, zwar erst abschließend, aber doch negativ ausfallen; oder sie können - wie bei Jean de Mandeville - von Anfang an die negative Dimension des Reiches anzeigen; die Texte können aber auch, wie das Land Lamori beispielhaft deutlich macht, Hinweise zur Einschätzung der Gemeinschaften geben, die unauflösbar widersprüchlich bleiben.

Außerdem können die Utopie-ähnlichen sozialen Einheiten in Bezug auf das, was die einzelnen Texte als bestehende Verhältnisse zu erkennen geben, verschiedene Funktionen erfüllen. Als Erzählsequenzen unter anderen sind die alternativen Gemeinschaften zwischen Episoden eingeordnet, in denen insbesondere vom Wunderbaren die Rede ist. In diesem Kontext liegt es nahe, dass staunen zu machen auch eine ihrer Funktionen ist. Eng verbunden sind explizit als wunderbar markierte Elemente und die Schilderung einer anderen Sozialordnung beispielsweise in den Ausführungen Jeans de Mandeville über das Reich des Presbyters Johannes. Dieser Bericht über das Priesterkönigreich legt nahe, dass auch die Auswirkungen besonderer Lebensbedingungen auf das Verhalten der Menschen zu den wunderbaren Elementen dieser Reiseschilderungen gezählt werden können. Mandevilles Ausführungen über das Reich der Brahmanen implizieren umgekehrt, dass sündloses Verhalten der Bewohner zum Wohlgefallen Gottes und in der Folge zu außergewöhnlichen Lebensbedingungen geführt habe; damit enthält der Text vermittelt durch göttliche Intervention den Gedanken, dass eine sozial anders geordnete, idealisierte Gemeinschaft machbar ist. Die hier vorgestellten Texte markieren aber auch, dass eine Distanzierung des Bestehenden bis hin zu ausdrücklicher Kritik daran Funktionen der Darstellung anderer und (zumindest in Teilen) idealisierter Ordnungen sein können. Die Bewohner des Landes Lamori in der Schilderung Odoricos da Pordenone sowie die der Insel Bragine bei Jean de Mandeville machen diese Kritik explizit. Auf der Grundlage eines breiteren Textkorpus müssen die hier angeführten Formen und Funktionen utopischer Elemente in der mittelalterlichen Reiseliteratur noch weiter ausdifferenziert und systematisiert werden, als diese Vorstellung der Texte und der Utopie-ähnlichen Reiche es leisten konnte.

Im Anschluss an die eingangs herausgestellte Bedeutung der gezielt unpräzisen Verortung der utopischen Insel in Thomas Morus' paradigmatischer Schrift für den frühneuzeitlichen Utopie-Begriff hat die Untersuchung alternativer Reiche in den drei mittelalterlichen Reiseschilderungen gezeigt, wie ungenau auch diese Gemeinschaften in der Regel verortet werden. Es ist allerdings in der mittelalterlichen Reiseliteratur kaum möglich, in ähnlicher Weise Fiktionalitätssignale zu identifizieren, wie ich sie einleitend in aller Kürze textimmanent für die Verortung der Insel Utopia zu zeigen versucht habe. Orte, die ex post als fiktiv klassifiziert werden können, und solche, die in hohem Maße an Faktisches gebunden sind, werden in diesen Texten nebeneinander behandelt, ${ }^{163}$ und die Formen ihrer

163 Hinsichtlich des unterschiedlichen Grades an Fiktionalität müssen auch die Utopie-ähnlichen Reiche selbst im Zuge einer detaillierten Analyse noch genauer differenziert werden. So kann man in systematischer Perspektive den Priesterkönig Johannes und sein Reich als fiktiv klassifizieren, dem Reich des Alten vom Berge wird man dagegen eine stärker referentielle Dimension zugestehen. 
Situierung unterscheiden sich nicht wesentlich. ${ }^{164}$ Das Verfahren der geographischen Verortung sozial anders geordneter Reiche in der mittelalterlichen Reiseliteratur deutet also darauf hin, dass diese Reiche keinen anderen epistemischen Status zugewiesen bekommen als andere ferne Länder. Anders als bei frühneuzeitlichen Utopien - für die hier beispielhaft Morus' ,Utopia' steht - geht die Inszenierung der Erreichbarkeit Utopie-ähnlicher Gemeinschaften in der mittelalterlichen Reiseliteratur nicht mit der Markierung ihrer Fiktionalität einher. Vielmehr scheinen diese Gemeinschaften auf Reisen besucht werden zu können. Wie andere Länder des Ostens sind sie sehr weit von Europa entfernt; man trifft lediglich seltener auf sie als auf andere entlegene Orte.

Der spezifische epistemische Status sozial anders geordneter Gemeinschaften der mittelalterlichen Reiseliteratur, auf den die Strategien der Verortung dieser sozialen Einheiten hindeuten, stellt für die Erforschung der Vorgeschichte frühneuzeitlicher Utopien eine besondere Herausforderung dar. Das gilt insbesondere deshalb, weil er mit einem umfassenden Wandel von Wissenskonzepten am Übergang vom Mittelalter zur Frühen Neuzeit zusammenhängt, der sich auch in dieser Gruppe von Texten zeigt. Mit einem stark vereinfachten historischen Narrativ gefasst transformiert sich Wissen, das in erster Linie durch Autoritäten abgesichert ist, zu Wissen, das auf Erfahrung basiert. ${ }^{165}$ Reisetexte des Mittelalters nehmen in der Regel für sich in Anspruch, authentisch zu sein, amalgamieren dabei aber zumeist wenige selbst gesehene Elemente mit Informationen aus Berichten anderer Reisender sowie mit zahlreichen Referenzen auf andere Formen tradierten Wissens, z. B. auf die Literatur über Alexander den Großen. ${ }^{166}$ Besonders die letztgenannten Anteile der Reiseberichte können häufig ex post als fiktiv charakterisiert werden, gelten aber historisch-zeitgenössisch als gesichertes Wissen. ${ }^{167}$ Daraus folgt weder, dass in mittelalterlichen Texten nicht unterschiedliche Grade von Fiktionalität unterschieden werden können ${ }^{168}$ noch dass nicht auch in Reiseberichten der Frühen Neuzeit die Grenze zwischen Faktualem und Fiktionalem zuweilen schwer zu ziehen ist oder gezielt unterminiert wird. ${ }^{169}$ Konsequenz dieser Beobachtung kann nur sein, dass in mittelalterlichen Reisetexten auf Erfah-

164 In der Mandeville-Übersetzung des Michel Velser beispielsweise wird auch Cayton, der Herrschaftssitz des Khans von Kathay, lediglich dadurch verortet, dass die Stadt am Weg liegt (vgl. Mandeville, Velser [Anm. 105], 129, 27-130, 2) - weder Entfernung noch Richtung zur vorausgegangenen Station werden angegeben. Cayton meint Taitu, die Residenz des Khan, unweit von Cambaluc (vgl. Pelliot [Anm. 63] Bd. 2, S. 843-845).

165 Vgl. Friederike Hassauer, Volkssprachliche Reiseliteratur. Faszination des Reisens und räumlicher ordo. In: Hans-Ulrich Gumbrecht, Ursula Link-Heer u. Peter-Michael Spangenberg (Hgg.), Grundriß der romanischen Literaturen des Mittelalters. Bd. 9/1. La littérature historiographique des origines à 1500. Heidelberg 1986, S. 259-283, hier S. 264, 267, 269-271. Zur Problematisierung sowohl des wechselseitigen Ausschlusses der Begriffe Tradition und Erfahrung bei der Konstitution von Wissen über die Fremde als auch der These einer linearen Entwicklung hin zur empirischen Begründung dieses Wissens in der Frühen Neuzeit vgl. Münkler (Anm. 55), S. 230-236.

166 Vgl. Wolf (Anm. 51), S. VIII; Simek (Anm. 79), S. 82 f.

167 Vgl. Peter Johanek, Weltbild und Literatur. Fiktive Geographie um 1300. In: Moraw, Peter (Hg.), Das geographische Weltbild um 1300. Politik im Spannungsfeld von Wissen, Mythos und Fiktion. Berlin 1989, S. $97-108$, hier S. 98 f.

168 Vgl. Jan-Dirk Müller, Literarische und andere Spiele. Zum Fiktionalitätsproblem in vormoderner Literatur. Poetica 36 (2004), S. $281-311$, hier S. 295.

169 Vgl. Justin Stagl, Eine Geschichte der Neugier. Die Kunst des Reisens 1550-1800. Wien u. a. 2002, S. 234-251. 
rung beruhendes Wissen in der Regel nicht in einer Weise in Konkurrenz zu tradiertem Wissen tritt, die dazu führt, dieses Wissen durchweg zu kritisieren oder gar zu verabschieden. ${ }^{170}$ Das Verhältnis beider Wissensformen wird also nicht als wechselseitiger Ausschluss verstanden, bei dem die Erfahrung grundsätzliche Priorität erhält. Das beschriebene Merkmal mittelalterlicher Reisetexte schließt ein, dass einzelne Korrekturen am tradierten Wissen auf der Grundlage autoptischer Erfahrung vorgenommen werden können. Beispiele für unterschiedliche Formen der Beglaubigung der vorgeführten Wissensbestände liefern bereits die einleitenden Passagen der hier besprochenen Reisetexte: Die deutsche Fassung von Marco Polos Bericht beruft sich allein auf Augenzeugenschaft; in der Übersetzung des Berichts von Odorico da Pordenone wird die Darstellung neben unmittelbaren Augenzeugen auch auf als verlässlich eingeschätzte Berichte anderer Augenzeugen und auf tradiertes Wissen gestützt. Mit der Aufforderung an die Rezipienten, die gegebenen Informationen selbst zu prüfen, zieht Mandevilles Text die eigene Verlässlichkeit, die durch Augenzeugenschaft beglaubigt wird, in Zweifel und weist damit auf den Wandlungsprozess der Ablösung von tradiertem und textuell verfasstem Wissen durch eine spezifische Vorstellung von Empirie voraus, der sich im Laufe des 16. und 17. Jahrhunderts vollzieht. ${ }^{171}$

Die Ausführungen zum epistemischen Status mittelalterlicher Vorformen von FerneUtopien sowie zum wissenshistorischen Veränderungsprozess, der den Horizont für die epistemische Charakterisierung von frühneuzeitlichen Utopien und mittelalterlichen Utopieähnlichen Reichen bildet, können an dieser Stelle nur vorläufig bleiben. Um den Zusammenhang zwischen beiden genauer bestimmen zu können und um Übergangsformen zwischen den beschriebenen epistemischen Status im Zuge des umrissenen Wandels von Utopien vom Mittelalter zur Frühen Neuzeit herausarbeiten zu können, ist ein deutlich breiteres Textkorpus notwendig als dieser Skizze zugrunde liegt. Ein erweitertes Korpus sollte insbesondere auch solche mittelalterlichen Texte einschließen, die das Thema der Reise nach Osten in stärker literarisierter Form aufgreifen als die hier verhandelte Reiseliteratur. Auf diese Weise wird sich das Bild von den mittelalterlichen Utopie-ähnlichen Reichen anhand gradueller Differenzen von Fiktionalität weiter ausdifferenzieren lassen.

\author{
Dr. Tilo Renz \\ Freie Universität Berlin \\ Institut für Deutsche und Niederländische Philologie \\ SFB 980 „Episteme in Bewegung“ \\ Habelschwerdter Allee 45 \\ 14195 Berlin \\ t.renz@fu-berlin.de
}

\footnotetext{
170 Vgl. Hassauer (Anm. 165), S. 270 f. Münkler hat diese generalisierende Einschätzung differenziert: Seit den Reisen der Franziskaner im 13. Jh. wird das tradierte Wissen durch Augenzeugenberichte korrigiert; durch diese Korrekturen sei es allerdings nicht zu einem Bruch zwischen tradiertem Wissen und Erfahrung gekommen (Münkler [Anm. 55], S. 274 f., 277).

171 Vgl. Münkler (Anm. 55), S. 279-282.
} 